\title{
A general version of the Hartogs extension theorem for separately holomorphic mappings between complex analytic spaces
}

\author{
VIÊT-ANH NGUYÊN
}

\begin{abstract}
Using recent development in Poletsky theory of discs, we prove the following result: Let $X, Y$ be two complex manifolds, let $Z$ be a complex analytic space which possesses the Hartogs extension property, let $A$ (resp. $B$ ) be a non locally pluripolar subset of $X$ (resp. $Y$ ). We show that every separately holomorphic mapping $f: W:=(A \times Y) \cup(X \times B) \longrightarrow Z$ extends to a holomorphic mapping $\hat{f}$ on $\widehat{W}:=\{(z, w) \in X \times Y: \widetilde{\omega}(z, A, X)+\widetilde{\omega}(w, B, Y)<1\}$ such that $\hat{f}=f$ on $W \cap \widehat{W}$, where $\widetilde{\omega}(\cdot, A, X)$ (resp. $\widetilde{\omega}(\cdot, B, Y))$ is the plurisubharmonic measure of $A$ (resp. $B$ ) relative to $X$ (resp. $Y$ ). Generalizations of this result for an $\mathrm{N}$-fold cross are also given.
\end{abstract}

Mathematics Subject Classification (2000): 32D15 (primary); 32D10 (secondary).

\section{Introduction}

The main purpose of this article is to give a general version of the well-known Hartogs extension theorem for separately holomorphic functions (see [8]). This theorem has been a source of inspiration for numerous research works in Complex Analysis for many years. It has developed into the beautiful and very active theory of separately analytic mappings. Nowadays, one finds a close connection between this theory and many other fields in Mathematics such as (Pluri)potential Theory, Partial Differential Equations and Theoretical Physics, etc. The recent survey articles by Nguyên Thanh Vân [18] and Peter Pflug [25] not only retrace the historic development, but also give some insights into the new research trends in this subject. Here we recall briefly the main steps in developing the theory of separately holomorphic mappings.

Very longtime after the ground-breaking work of Hartogs, the subject was reborn, around the years 50-60s, thanks to the Japanese school (see [35], [38] and the references therein). However, an important impetus was only made by Siciak in the works [36, 37], where he established some significant generalizations of the Hartogs extension theorem. According to Siciak's general formulation of this theorem, the 
problem is to determine the envelope of holomorphy for separately holomorphic functions defined on some cross sets. The theorems obtained under this formulation are often called cross theorems. Using the so-called relative extremal function, Siciak completed the problem for the case where the cross set consists of a product of domains in $\mathbb{C}$.

The next deep steps were initiated by Zahariuta in 1976 (see [39]) when he started to use the method of common bases of Hilbert spaces. This original approach permits him to obtain new cross theorems for some cases where the cross consists of a product of Stein manifolds. As a consequence, he was able to generalize the result of Siciak in higher dimensions.

Later, Nguyên Thanh Vân and Zeriahi (see [22, 23, 24]) developed the method of doubly orthogonal bases of Bergman type in order to generalize the result of Zahariuta. This is a significantly simpler and more constructive version of Zahariuta's original method. Nguyên Thanh Vân and Zeriahi have recently achieved an elegant improvement of their method (see [19], [40]).

Using the method of Siciak, Shiffman (see [34]) was the first to generalize some results of Siciak to separately holomorphic mappings with values in a complex analytic space.

The most general result to date is contained in a recent work by Alehyane and Zeriahi (see Theorem 2.2.4 in [3]). Namely, they are able to define the envelope of holomorphy of any cross of a product of subdomains of Stein manifolds in terms of the plurisubharmonic measure.

In this work we generalize, in some sense, the result of Alehyane-Zeriahi to any cross of a product of arbitrary complex manifolds. The main ingredient in our approach is Poletsky theory of discs developed in [26, 27], Rosay's Theorem on holomorphic discs (see [32]) and Alehyane-Zeriahi Theorem (see [3]). Another important technique is to use level sets of the plurisubharmonic measure. This technique was originally introduced in a recent joint-work of Pflug and the author (see [28]). However, it appears to be very successful in solving many problems arising from the theory of separately holomorphic and meromorphic mappings.

This paper is organized as follows.

In Section 2, after introducing some terminology and notation, we recall Alehyane-Zeriahi Theorem and state our main result.

The tools which are needed for the proof of the main result are developed in Sections 3.

The proof of the main result for the case of an 2-fold cross is divided into three parts, which correspond to Sections 4, 5, and 6.

The general case is treated in Section 7. Finally, we conclude the article with some remarks and open questions.

The theory of separately holomorphic and meromorphic mappings has received much attention in the past few years. We only mention here some directions of the current research. Separate analyticity in infinite dimension is growing quite rapidly since the work of Noverraz [20]. Many results in this direction are obtained by Nguyên Van Khuê, Nguyên Thanh Vân and their co-workers (see the discussion in [16] and [18]). On the other hand, the recent development also focuses on cross theorems with pluripolar singularities and boundary cross theorems. For the latest 
results as well as a comprehensive introduction to the latter two directions, the reader may consult some works of Jarnicki and Pflug in $[12,13,14]$ and recent articles of Pflug and the author (see [28, 29]).

ACKNOWLEDGEMEnTs. The paper was written while the author was visiting the Carl von Ossietzky Universität Oldenburg being supported by The Alexander von Humboldt Foundation. He wishes to express his gratitude to these organizations. He also would like to thank Professor Peter Pflug for very stimulating discussions, and the referee for many valuable remarks.

\section{Preliminaries and statement of the main result}

In order to recall the classical cross theorem and to state the main result, we need to introduce some notation and terminology. In fact, we keep the main notation from the works in [11], [33].

\subsection{Local pluripolarity and plurisubharmonic measure, cross and separate holomorphicity}

In the sequel, all complex manifolds are supposed to be of finite local dimension (i.e. the dimension of any connected component of the manifold is finite), and all complex analytic spaces considered in this work are supposed to be reduced, irreducible and of finite dimension.

Let $\mathcal{M}$ be a complex manifold and let $A$ be a subset of $\mathcal{M}$. Put

$$
h_{A, \mathcal{M}}:=\sup \{u: u \in \mathcal{P} \mathcal{S H}(\mathcal{M}), u \leq 1 \text { on } \mathcal{M}, u \leq 0 \text { on } A\} \text {, }
$$

where $\mathcal{P} \mathcal{S H}(\mathcal{M})$ denotes the cone of all plurisubharmonic functions on $\mathcal{M}$.

$A$ is said to be pluripolar in $\mathcal{M}$ if there is $u \in \mathcal{P S H}(\mathcal{M})$ such that $u$ is not identically $-\infty$ on every connected component of $\mathcal{M}$ and $A \subset\{z \in \mathcal{M}: u(z)=-\infty\}$. $A$ is said to be locally pluripolar in $\mathcal{M}$ if for any $z \in A$, there is an open neighborhood $V$ of $z$ such that $A \cap V$ is pluripolar in $V$. A is said to be nonpluripolar (resp. non locally pluripolar) if it is not pluripolar (resp. not locally pluripolar). According to a classical result of Josefson and Bedford (see [9], [4]), if $\mathcal{M}$ is a Riemann domain over a Stein manifold, then $A \subset \mathcal{M}$ is locally pluripolar if and only if it is pluripolar.

In the sequel, for a function $h: \mathcal{M} \longrightarrow \mathbb{R}$, its upper semicontinuous regularization $h^{*}: \mathcal{M} \longrightarrow \mathbb{R}$ is defined by

$$
h^{*}(z):=\limsup _{w \rightarrow z} h(w), \quad z \in \mathcal{M} .
$$

Next, we say that a set $A \subset \mathcal{M}$ is locally pluriregular at a point $a \in \bar{A}$ if $h_{A \cap U, U}^{*}(a)=0$ for all open neighborhoods $U$ of $a$. Moreover, $A$ is said to be 
locally pluriregular if it is locally pluriregular at every point $a \in A$. We denote by $A^{*}=A_{\mathcal{M}}^{*}$ the set of all points $a \in \bar{A}$ at which $A$ is locally pluriregular. If $A$ is non locally pluripolar, then a classical result of Bedford and Taylor (see $[4,5])$ says that $A^{*}$ is non locally pluripolar and $A \backslash A^{*}$ is locally pluripolar. Moreover, $A^{*}$ is locally of type $\mathcal{G}_{\delta}$ (i.e. for every $a \in A^{*}$, there is an open neighborhood $U$ of $a$ such that $A^{*} \cap U$ is a countable intersection of open sets), and $A^{*}$ is locally pluriregular (i.e. $\left.\left(A^{*}\right)^{*}=A^{*}\right)$.

The plurisubharmonic measure of $A$ relative to $\mathcal{M}$ is the function ${ }^{(1)}$ defined by

$$
\widetilde{\omega}(z, A, \mathcal{M}):=h_{A^{*}, \Omega}^{*}(z), \quad z \in \mathcal{M} .
$$

Observe that $\widetilde{\omega}(\cdot, A, \mathcal{M}) \in \mathcal{P} \mathcal{S H}(\mathcal{M})$ and $0 \leq \widetilde{\omega}(z, A, \mathcal{M}) \leq 1, z \in \mathcal{M}$.

We shall show in Proposition 3.6 below that if $\mathcal{M}$ is a subdomain of a Stein manifold, then the above definition coincides with the one given by formula (2.1.2) in Alehyane-Zeriahi's article [3]. For a good background of the pluripotential theory, see the books [11] or [15].

Let $N \in \mathbb{N}, N \geq 2$, and let $\varnothing \neq A_{j} \subset D_{j}$, where $D_{j}$ is a complex manifold, $j=1, \ldots, N$. We define an $N$-fold cross

$$
\begin{aligned}
X & :=\mathbb{X}\left(A_{1}, \ldots, A_{N} ; D_{1}, \ldots, D_{N}\right) \\
& :=\bigcup_{j=1}^{N} A_{1} \times \cdots \times A_{j-1} \times D_{j} \times A_{j+1} \times \cdots A_{N} .
\end{aligned}
$$

Following a terminology of Alehyane-Zeriahi [3], we define the regular part $X^{*}$ of $X$ as follows

$$
\begin{aligned}
X^{*} & =\mathbb{X}^{*}\left(A_{1}, \ldots, A_{N} ; D_{1}, \ldots, D_{N}\right):=\mathbb{X}\left(A_{1}^{*}, \ldots, A_{N}^{*} ; D_{1}, \ldots, D_{N}\right) \\
& =\bigcup_{j=1}^{N} A_{1}^{*} \times \cdots \times A_{j-1}^{*} \times D_{j} \times A_{j+1}^{*} \times \cdots A_{N}^{*} .
\end{aligned}
$$

Moreover, put

$$
\omega(z):=\sum_{j=1}^{N} \widetilde{\omega}\left(z_{j}, A_{j}, D_{j}\right), \quad z=\left(z_{1}, \ldots, z_{N}\right) \in D_{1} \times \cdots \times D_{N} .
$$

For an $N$-fold cross $X:=\mathbb{X}\left(A_{1}, \ldots, A_{N} ; D_{1}, \ldots, D_{N}\right)$ let

$$
\widehat{X}=\widehat{\mathbb{X}}\left(A_{1}, \ldots, A_{N} ; D_{1}, \ldots, D_{N}\right):=\left\{\left(z_{1}, \ldots, z_{N}\right) \in D_{1} \times \cdots \times D_{N}: \omega(z)<1\right\} \text {. }
$$

It is not difficult to see that $X^{*} \subset \widehat{X}$.

${ }^{(1)}$ The notation $\omega(\cdot, A, \mathcal{M})$ is historically reserved for the relative extremal function. The latter function is defined by

$$
\omega(z, A, \mathcal{M}):=h_{A, \Omega}^{*}(z), \quad z \in \mathcal{M} .
$$

An example in [1] shows that in general, $\omega(\cdot, A, \mathcal{M}) \neq \widetilde{\omega}(\cdot, A, \mathcal{M})$. 
Let $Z$ be a complex analytic space. We say that a mapping $f: X \longrightarrow Z$ is separately holomorphic and write $f \in \mathcal{O}_{S}(X, Z)$, if for any $j \in\{1, \ldots, N\}$ and $\left(a^{\prime}, a^{\prime \prime}\right) \in\left(A_{1} \times \cdots \times A_{j-1}\right) \times\left(A_{j+1} \times \cdots \times A_{N}\right)$ the restricted mapping $\left.f\left(a^{\prime}, \cdot, a^{\prime \prime}\right)\right|_{D_{j}}$ is holomorphic on $D_{j}$.

Throughout the paper, for a function $f: M \longrightarrow \mathbb{C}$, let $|f|_{M}$ denote $\sup _{M}|f|$. Finally, for a complex manifold $\mathcal{M}$ and a complex analytic space $Z$, let $\mathcal{O}(\mathcal{M}, Z)$ denote the set of all holomorphic mappings from $\mathcal{M}$ to $Z$.

\subsection{Hartogs extension property}

We recall here the following notion introduced by Shiffman [34]. Let $p \geq 2$ be an integer. For $0<r<1$, the Hartogs figure in dimension $p$, denoted by $H_{p}(r)$, is given by

$$
H_{p}(r):=\left\{\left(z^{\prime}, z_{p}\right) \in E^{p}:\left\|z^{\prime}\right\|<r \text { or }\left|z_{p}\right|>1-r\right\},
$$

where $E$ is the unit disc of $\mathbb{C}$ and $z^{\prime}=\left(z_{1}, \ldots, z_{p-1}\right),\left\|z^{\prime}\right\|:=\max _{1 \leq j \leq p-1}\left|z_{j}\right|$.

Definition 2.1. A complex analytic space $Z$ is said to possess the Hartogs extension property in dimension $p$ if $Z$ has a countable bases of open subsets, and every mapping $f \in \mathcal{O}\left(H_{p}(r), Z\right)$ extends to a mapping $\hat{f} \in \mathcal{O}\left(E^{p}, Z\right)$. Moreover, $Z$ is said to possess the Hartogs extension property if it does in any dimension $p \geq 2$.

It is a classical result of Ivashkovich (see [10]) that if $Z$ possesses the Hartogs extension property in dimension 2 , then it does in all dimensions $p \geq 2$. Some typical examples of complex analytic spaces possessing the Hartogs extension property are the complex Lie groups (see [2]), the taut spaces (see [41]), the Hermitian manifold with negative holomorphic sectional curvature (see [34]), the holomorphically convex Kähler manifold without rational curves (see [10]).

Here we mention an important characterization due to Shiffman (see [34]).

Theorem 2.2. A complex analytic space $Z$ possesses the Hartogs extension property if and only if for every domain $D$ of any Stein manifold $\mathcal{M}$, every mapping $f \in \mathcal{O}(D, Z)$ extends to a mapping $\hat{f} \in \mathcal{O}(\widehat{D}, Z)$, where $\widehat{D}$ is the envelope of holomorphy of $D$.

\subsection{Motivations for our work}

We are now able to formulate what we will quote in the sequel as the classical cross theorem.

Theorem 2.3. (Alehyane-Zeriahi [3, Theorem 2.2.4]) Let $X_{j}$ be a Stein manifold, let $D_{j} \subset X_{j}$ be a domain and $A_{j} \subset D_{j}$ a nonpluripolar subset, $j=1, \ldots, N$. Let $Z$ be a complex analytic space possessing the Hartogs extension property. Then for any mapping $f \in \mathcal{O}_{s}(X, Z)$, there is a unique mapping $\hat{f} \in \mathcal{O}(\widehat{X}, Z)$ such that $\hat{f}=f$ on $X \cap \widehat{X}$. 
The following example given by Alehyane-Zeriahi (see [3]) shows that the hypothesis on $Z$ is necessary. Consider the mapping $f: \mathbb{C}^{2} \longrightarrow \mathbb{P}^{1}$ given by

$$
f(z, w):= \begin{cases}{\left[(z+w)^{2}:(z-w)^{2}\right],} & (z, w) \neq(0,0) \\ {[1: 1],} & (z, w)=(0,0) .\end{cases}
$$

Then $f \in \mathcal{O}_{s}\left(\mathbb{X}(\mathbb{C}, \mathbb{C} ; \mathbb{C}, \mathbb{C}), \mathbb{P}^{1}\right)$, but $f$ is not continuous at $(0,0)$.

The question naturally arises whether Theorem 2.3 is still true if $D_{j}$ is not necessarily a subdomain of a Stein manifold, $j=1, \ldots, N$.

\subsection{Statement of the main results and outline of the proofs}

We are now ready to state the main results.

Theorem A. Let $D_{j}$ be a complex manifold and $A_{j} \subset D_{j}$ a non locally pluripolar subset, $j=1, \ldots, N$. Let $Z$ be a complex analytic space possessing the Hartogs extension property. Then for any mapping $f \in \mathcal{O}_{s}(X, Z)$, there is a unique mapping $\hat{f} \in \mathcal{O}(\widehat{X}, Z)$ such that $\hat{f}=f$ on $X \cap \widehat{X}$. If, moreover, $Z=\mathbb{C}$ and $|f|_{X}<\infty$, then

$$
|\hat{f}(z)| \leq|f|_{A}^{1-\omega(z)}|f|_{X}^{\omega(z)}, \quad z \in \widehat{X}
$$

In virtue of a theorem of Josefson and Bedford (see Subsection 2.1 above), the classical cross theorem is an immediate consequence of Theorem A.

Theorem A has an important corollary. Before stating this, we need to introduce a terminology. A complex manifold $\mathcal{M}$ is said to be a Liouville manifold if $\mathcal{P S H}(\mathcal{M})$ does not contain any non-constant bounded above functions. We see clearly that the class of Liouville manifolds contains the class of connected compact manifolds.

Corollary B. Let $D_{j}$ be a complex manifold and $A_{j} \subset D_{j}$ a non locally pluripolar subset, $j=1, \ldots, N$. Let $Z$ be a complex analytic space possessing the Hartogs extension property. Suppose in addition that $D_{j}$ is a Liouville manifold, $j=2, \ldots, N$. Then for any mapping $f \in \mathcal{O}_{S}(X, Z)$, there is a unique mapping $\hat{f} \in \mathcal{O}\left(D_{1} \times \cdots \times D_{N}, Z\right)$ such that $\hat{f}=f$ on $X$.

Corollary B follows immediately from Theorem A since $\widetilde{\omega}\left(\cdot, A_{j}, D_{j}\right) \equiv 0, j=$ $2, \ldots, N$.

We give below some ideas of the proof of Theorem A.

Our method consists in two steps. In the first step, we investigate the special case where each $A_{j}$ is an open set, $j=1, \ldots, N$. In the second one, we treat the general case.

In order to carry out the first step, we apply Poletsky theory of discs and Rosay's Theorem on holomorphic discs (see Theorem 3.1 below). Consequently, 
we may construct an extension mapping $\hat{f}$ on $\widehat{X}$. To prove that $\hat{f}$ is holomorphic, we appeal to the classical cross theorem (Theorem 2.3).

In the second step we reduce the general situation to the above special case. The key technique is to use level sets of the plurisubharmonic measure. More precisely, we exhaust each $D_{j}$ by the level sets of the plurisubharmonic measure $\widetilde{\omega}\left(\cdot, A_{j}, D_{j}\right)$, i.e. by $D_{j, \delta}:=\left\{z_{j} \in D_{j}: \widetilde{\omega}\left(z_{j}, A_{j}, D_{j}\right)<1-\delta\right\}(0<\delta<1)$. We replace in the same way the set $A_{j}$ by an open set $A_{j, \delta}$ such that $\widetilde{\omega}\left(\cdot, A_{j, \delta}, D_{j, \delta}\right)$ behaves, in some sense, like $\widetilde{\omega}\left(\cdot, A_{j}, D_{j}\right)$ as $\delta \rightarrow 0^{+}$.

Applying Theorem 2.3 locally and making an intensive use of Theorem 3.1, we can propagate the separate holomorphicity of $f$ to a mapping $\tilde{f}_{\delta}$ defined on the cross $X_{\delta}:=\mathbb{X}\left(A_{1, \delta}, \ldots, A_{N, \delta} ; D_{1, \delta}, \ldots, D_{N, \delta}\right)$. Consequently, the first step applies and one obtains a mapping $\hat{f}_{\delta} \in \mathcal{O}\left(\widehat{X_{\delta}}, Z\right)$. Gluing the family $\left(\hat{f}_{\delta}\right)_{0<\delta<1}$, we obtain the desired extension mapping $\hat{f}$.

\section{Preparatory results}

We recall here the auxiliary results and some background of the pluripotential theory needed for the proof of Theorem A.

\subsection{Poletsky theory of discs and Rosay's Theorem on holomorphic dises}

Let $E$ denote as usual the unit disc in $\mathbb{C}$. For a complex manifold $\mathcal{M}$, let $\mathcal{O}(\bar{E}, \mathcal{M})$ denote the set of all holomorphic mappings $\phi: E \longrightarrow \mathcal{M}$ which extend holomorphically to a neighborhood of $\bar{E}$. Such a mapping $\phi$ is called a holomorphic disc on $\mathcal{M}$. Moreover, for a subset $A$ of $\mathcal{M}$, let

$$
1_{A}(z):= \begin{cases}1, & z \in A \\ 0, & z \in \mathcal{M} \backslash A .\end{cases}
$$

In the work [32] Rosay proved the following remarkable result.

Theorem 3.1. Let $u$ be an upper semicontinuous function on a complex manifold $\mathcal{M}$. Then the Poisson functional of $u$ defined by

$$
\mathcal{P}[u](z):=\inf \left\{\frac{1}{2 \pi} \int_{0}^{2 \pi} u\left(\phi\left(e^{i \theta}\right)\right) d \theta: \phi \in \mathcal{O}(\bar{E}, \mathcal{M}), \phi(0)=z\right\},
$$

is plurisubharmonic on $\mathcal{M}$.

Rosay's Theorem may be viewed as an important development in Poletsky theory of discs. Observe that special cases of Theorem 3.1 have been considered by Poletsky (see [26, 27]), Lárusson-Sigurdsson (see [17]) and Edigarian (see [6]).

We also need the following result (see [32, Lemmas 1.1 and 1.2]). 
Lemma 3.2. Let $\mathcal{M}$ be a complex manifold and let $A$ be a nonempty open subset of $\mathcal{M}$. Then for any $\epsilon>0$ and any $z_{0} \in \mathcal{M}$, there are an open neighborhood $U$ of $z_{0}$, an open subset $T$ of $\mathbb{C}$, and a family of holomorphic discs $\left(\phi_{z}\right)_{z \in U} \subset \mathcal{O}(\bar{E}, \mathcal{M})$ with the following properties:

(i) $\Phi \in \mathcal{O}(U \times E, \mathcal{M})$, where $\Phi(z, t):=\phi_{z}(t), \quad(z, t) \in U \times E$;

(ii) $\phi_{z}(0)=z, \quad z \in U$;

(iii) $\phi_{z}(t) \in A, \quad t \in T \cap \bar{E}, z \in U$;

(iv) $\frac{1}{2 \pi} \int_{0}^{2 \pi} 1_{\partial E \backslash T}\left(e^{i \theta}\right) d \theta<\mathcal{P}\left[1_{\mathcal{M} \backslash A}\right]\left(z_{0}\right)+\epsilon$.

Proof. For any $\rho>0$, let $E_{\rho}$ denote the disc $\{t \in \mathbb{C}:|t|<\rho\}$. Fix an arbitrary point $z_{0} \in \mathcal{M}$. We apply Theorem 3.1 to the upper semicontinuous function $1_{\mathcal{M} \backslash A}$. Consequently, for any $\epsilon>0$, one may find an $r>1$ and a holomorphic mapping $\phi \in \mathcal{O}\left(E_{r}, \mathcal{M}\right)$ such that

$$
\phi(0)=z_{0} \quad \text { and } \quad \frac{1}{2 \pi} \int_{0}^{2 \pi} 1_{\mathcal{M} \backslash A}\left(\phi\left(e^{i \theta}\right)\right) d \theta<\mathcal{P}\left[1_{\mathcal{M} \backslash A}\right]\left(z_{0}\right)+\frac{\epsilon}{2} .
$$

Consider the embedding $\tau: E_{r} \longrightarrow \mathbb{C} \times \mathcal{M}$ given by $\tau(t):=(t, \phi(t)), t \in E_{r}$. Then the image $\tau\left(E_{r}\right)$ is a Stein submanifold of $\mathbb{C} \times \mathcal{M}$. Fix any $\tilde{r}$ such that $1<$ $\tilde{r}<r$ and let $d$ be the dimension of the connected component of $\mathcal{M}$ containing $z_{0}$. By Lemma 1.1 in [32], there is an injective holomorphic mapping $\tilde{\tau}: E_{\tilde{r}}^{d+1} \longrightarrow$ $\mathbb{C} \times \mathcal{M}$ such that $\tilde{\tau}(t, 0)=\tau(t)=(t, \phi(t)),|t|<\tilde{r}$. Let $\Pi$ be the canonical projection from $\mathbb{C} \times \mathcal{M}$ onto $\mathcal{M}$. Then there are a sufficiently small neighborhood $U$ of $z_{0}$ and a real number $\rho: 1<\rho<\tilde{r}$ such that, for every $z \in U$, the mapping $\phi_{z}: E_{\rho} \longrightarrow \mathcal{M}$ given by

$$
\phi_{z}(t):=\Pi \circ \tilde{\tau}\left((t, 0)+\tilde{\tau}^{-1}(0, z)\right), \quad t \in E_{\rho},
$$

is holomorphic.

Using the explicit formula (3.2), assertion (i) follows. Moreover, $\phi_{z}(0)=$ $\Pi(0, z)=z$ for $z \in U$, which proves assertion (ii). In addition,

$$
\phi_{z_{0}}(t)=(\Pi \circ \tilde{\tau})(t, 0)=\phi(t) .
$$

In virtue of (3.2), observe that as $z$ approaches $z_{0}$ in $U, \phi_{z}$ converges uniformly to $\phi_{z_{0}}$ on $\bar{E}$. Consequently, by shrinking $U$ if necessary, we may find an open subset $T$ of the open set $\left\{t \in E_{\rho}: \phi_{z_{0}}(t) \in A\right\}$ such that assertion (iii) is fulfilled and

$$
\frac{1}{2 \pi} \int_{0}^{2 \pi} 1_{\partial E \backslash T}\left(e^{i \theta}\right) d \theta<\frac{1}{2 \pi} \int_{0}^{2 \pi} 1_{\mathcal{M} \backslash A}\left(\phi_{z_{0}}\left(e^{i \theta}\right)\right) d \theta+\frac{\epsilon}{2} .
$$

This, combined with the estimate in (3.1) and (3.3), implies assertion (iv). Hence, the proof of the lemma is complete. 


\subsection{The plurisubharmonic measure and its level sets}

We begin this subsection with the following simple but very useful result.

Lemma 3.3. Let $T$ be an open subset of $\bar{E}$. Then

$$
\widetilde{\omega}(0, T \cap E, E) \leq \frac{1}{2 \pi} \int_{0}^{2 \pi} 1_{\partial E \backslash T}\left(e^{i \theta}\right) d \theta .
$$

Proof. Observe that, by definition,

$$
\widetilde{\omega}(t, T \cap E, E) \leq \omega_{E}(t, T \cap \partial E), \quad t \in E,
$$

where $\omega_{E}(t, T \cap \partial E)$ is the harmonic measure for $E$ (see [31, p. 96]). Since

$$
\omega_{E}(0, T \cap \partial E)=\frac{1}{2 \pi} \int_{0}^{2 \pi} 1_{\partial E \backslash T}\left(e^{i \theta}\right) d \theta,
$$

the desired conclusion follows from the above estimate.

Proposition 3.4. Let $\mathcal{M}$ be a complex manifold and $A$ a nonempty open subset of $\mathcal{M}$. Then $\tilde{\omega}(z, A, \mathcal{M})=\mathcal{P}\left[1_{\mathcal{M} \backslash A}\right](z), z \in \mathcal{M}$.

Proof. First, since $A$ is open, it is clear that $A^{*}=A$. In addition, applying Theorem 3.1 to $1_{\mathcal{M} \backslash A}$ and using the explicit formula of $\mathcal{P}\left[1_{\mathcal{M} \backslash A}\right]$, we see that $\mathcal{P}\left[1_{\mathcal{M} \backslash A}\right] \in$ $\mathcal{P S H}(\mathcal{M}), \mathcal{P}\left[1_{\mathcal{M} \backslash A}\right] \leq 1$ and $\mathcal{P}\left[1_{\mathcal{M} \backslash A}\right](z)=0, z \in A$. Consequently,

$$
\mathcal{P}\left[1_{\mathcal{M} \backslash A}\right](z) \leq \widetilde{\omega}(z, A, \mathcal{M}), \quad z \in \mathcal{M}
$$

To see the opposite inequality, let $u \in \mathcal{P} \mathcal{S H}(\mathcal{M})$ such that $u \leq 1$ and $u(z) \leq 0$, $z \in A$. For any point $z_{0} \in \mathcal{M}$ and any $\epsilon>0$, by Theorem 3.1, there is a holomorphic disc $\phi \in \mathcal{O}(\bar{E}, \mathcal{M})$ such that

$$
\phi(0)=z_{0} \quad \text { and } \quad \frac{1}{2 \pi} \int_{0}^{2 \pi} 1_{\mathcal{M} \backslash A}\left(\phi\left(e^{i \theta}\right)\right) d \theta<\mathcal{P}\left[1_{\mathcal{M} \backslash A}\right]\left(z_{0}\right)+\epsilon .
$$

Consequently, by setting $\phi^{-1}(A):=\{t \in \bar{E}: \phi(t) \in A\}$, we obtain

$$
u\left(z_{0}\right)=(u \circ \phi)(0) \leq \widetilde{\omega}\left(0, \phi^{-1}(A), E\right) \leq \frac{1}{2 \pi} \int_{0}^{2 \pi} 1_{\mathcal{M} \backslash A}\left(\phi\left(e^{i \theta}\right)\right) d \theta
$$

where the first estimate is trivial and the second one follows from Lemma 3.3. This, combined with (3.4), implies that $u\left(z_{0}\right)<\mathcal{P}\left[1_{\mathcal{M} \backslash A}\right]\left(z_{0}\right)+\epsilon$. Since $u$, $\epsilon$ and $z_{0}$ are arbitrarily chosen, we conclude that $\widetilde{\omega}(z, A, \mathcal{M}) \leq \mathcal{P}\left[1_{\mathcal{M} \backslash A}\right](z), z \in \mathcal{M}$. This completes the proof. 
Proposition 3.5. Let $\mathcal{M}$ be a complex manifold and $A$ a non locally pluripolar subset of $\mathcal{M}$. For $0<\epsilon<1$, define the " $\epsilon$-level set of $\mathcal{M}$ relative to $A$ " as follows

$$
\mathcal{M}_{\epsilon, A}:=\{z \in \mathcal{M}: \widetilde{\omega}(z, A, \mathcal{M})<1-\epsilon\} .
$$

Then:

1) For every locally pluripolar subset $P$ of $\mathcal{M},(A \cup P)^{*}=A^{*}$ and $\widetilde{\omega}(\cdot, A \cup$ $P, \mathcal{M})=\widetilde{\omega}(\cdot, A, \mathcal{M}) .\left(A^{*}\right)^{*}=A^{*}$. If, moreover, $A$ is open, then $A^{*}=A$.

2) Let $\mathcal{N}$ be an open subset of $\mathcal{M}$ and $B \subset A \cap \mathcal{N}$. Then $\widetilde{\omega}(z, A, \mathcal{M}) \leq \widetilde{\omega}(z, B, \mathcal{N})$, $z \in \mathcal{N}$.

3) Let $\mathcal{N}$ be a connected component of $\mathcal{M}$, then $\widetilde{\omega}(z, A \cap \mathcal{N}, \mathcal{N})=\widetilde{\omega}(z, A, \mathcal{M})$, $z \in \mathcal{N}$.

4) $\widetilde{\omega}\left(z, A \cap A^{*}, \mathcal{M}_{\epsilon, A}\right)=\frac{\widetilde{\omega}(z, A, \mathcal{M})}{1-\epsilon}, z \in \mathcal{M}_{\epsilon, A}$.

5) Every connected component of $\mathcal{M}_{\epsilon, A}$ contains a non locally pluripolar subset of $A \cap A^{*}$. If, moreover, $A$ is open, then every connected component of $\mathcal{M}_{\epsilon, A}$ contains a nonempty open subset of $A$.

Proof. Part 1) is an immediate consequence of the following identity (see Lemma 3.5.3 in [11])

$$
h_{A \cup P, U}^{*}=h_{A, U}^{*},
$$

where $U$ is a bounded open subset of $\mathbb{C}^{n}, A$ and $P$ are subsets of $U$, and $P$ is pluripolar.

Part 2) and Part 3) are trivial using the definition of the plurisubharmonic measure.

Now we turn to Part 4). Observe that for any $a \in A^{*}$,

$$
\widetilde{\omega}(a, A, \mathcal{M})=\widetilde{\omega}\left(a, A^{*}, \mathcal{M}\right)=0,
$$

where the first equality follows from the definition of the plurisubharmonic measure, and the second one from Part 2) and the assumption that $a \in A^{*}$. Hence, $A^{*} \subset \mathcal{M}_{\epsilon, A}$. In addition, we have clearly that $\frac{\widetilde{\omega}(z, A, \mathcal{M})}{1-\epsilon} \leq 1, z \in \mathcal{M}_{\epsilon, A}$. This, combined with (3.5), implies that

$$
\frac{\widetilde{\omega}(z, A, \mathcal{M})}{1-\epsilon} \leq \widetilde{\omega}\left(z, A \cap A^{*}, \mathcal{M}_{\epsilon, A}\right), \quad z \in \mathcal{M}_{\epsilon, A} .
$$

To prove the converse inequality of (3.6), let $u \in \mathcal{P} \mathcal{S H}\left(\mathcal{M}_{\epsilon, A}\right)$ be such that $u \leq 1$ on $\mathcal{M}_{\epsilon, A}$ and $u \leq 0$ on $A^{*}$. Consider the following function

$$
\hat{u}(z):= \begin{cases}\max \{(1-\epsilon) u(z), \widetilde{\omega}(z, A, \mathcal{M})\}, & z \in \mathcal{M}_{\epsilon, A}, \\ \widetilde{\omega}(z, A, \mathcal{M}), & z \in \mathcal{M} \backslash \mathcal{M}_{\epsilon, A} .\end{cases}
$$

It can be checked that $\hat{u} \in \mathcal{P} \mathcal{S H}(\mathcal{M})$ and $\hat{u} \leq 1$. Moreover, in virtue of the assumption on $u$ and (3.5), we have that

$$
\hat{u}(a) \leq \max \{(1-\epsilon) u(a), \widetilde{\omega}(a, A, \mathcal{M})\}=0, \quad a \in A^{*} .
$$


Consequently, $\hat{u} \leq \widetilde{\omega}\left(\cdot, A^{*}, \mathcal{M}\right)=\widetilde{\omega}(\cdot, A, \mathcal{M})$. In particular, one gets that

$$
u(z) \leq \frac{\widetilde{\omega}(z, A, \mathcal{M})}{1-\epsilon}, \quad z \in \mathcal{M} .
$$

Since $u$ is arbitrary, we deduce from the latter estimate that the converse inequality of (3.6) also holds. This completes the proof of Part 4).

Part 5) follows immediately from Parts 3) and 4).

Hence, the proof of the proposition is finished.

The following result shows that our definition of the plurisubharmonic measure recovers the one given by Alehyane-Zeriahi in [3, formula (2.1.2)].

Proposition 3.6. Let $\mathcal{M}$ be a Stein manifold. Let $U$ be a subdomain of $\mathcal{M}$ which admits an exhaustive sequence of open subsets $\left(U_{j}\right)_{j=1}^{\infty}$, i.e. $U_{j} \Subset U_{j+1}$ and $\bigcup_{j=1}^{\infty} U_{j}=U$. Then for any subset $A \subset U$, there holds

$$
\widetilde{\omega}(z, A, U)=\lim _{j \rightarrow \infty} h_{A \cap U_{j}, U_{j}}^{*}(z), \quad z \in U .
$$

Proof. First observe that the sequence $\left(h_{A \cap U_{j}, U_{j}}^{*}\right)_{j=1}^{\infty}$ decreases, as $j \rightarrow \infty$, to a function $h \in \mathcal{P S H}(\mathcal{M})$.

Next, since $U_{j} \Subset \mathcal{M}$ and $A \backslash A^{*}$ is pluripolar, it follows from Lemma 2.2 in [1] and Part 1) of Proposition 3.5 that $h_{A \cap U_{j}, U_{j}}^{*}=h_{A \cap A^{*} \cap U_{j}, U_{j}}^{*}=\widetilde{\omega}\left(\cdot, A \cap U_{j}, U_{j}\right)$ for any $j \geq 1$. Consequently, applying Part 2) of Proposition 3.5 yields that

$$
\widetilde{\omega}(z, A, U) \leq \liminf _{j \rightarrow \infty} \widetilde{\omega}\left(z, A \cap U_{j}, U_{j}\right)=\lim _{j \rightarrow \infty} h_{A \cap U_{j}, U_{j}}^{*}(z)=h(z), \quad z \in U .
$$

On the other hand, using the above definition of $h$, one can check that $h \leq 1$ on $\mathcal{M}$ and $h \leq 0$ on $\bigcup_{j=1}^{\infty}\left(A \cap U_{j}\right)^{*}$. Since the latter union is equal to $A^{*}$, it follows that $h \leq \widetilde{\omega}(\cdot, A, U)$. This, combined with estimate (3.7), completes the proof.

Proposition 3.7. Let $\mathcal{M}_{j}$ be a complex manifold and $A_{j}$ a nonempty open subset of $\mathcal{M}_{j}, j=1, \ldots, N, N \geq 2$.

1) Then, for $z=\left(z_{1}, \ldots, z_{N}\right) \in \mathcal{M}_{1} \times \cdots \times \mathcal{M}_{N}$,

$$
\widetilde{\omega}\left(z, A_{1} \times \cdots \times A_{N}, \mathcal{M}_{1} \times \cdots \times \mathcal{M}_{N}\right)=\max _{j=1, \ldots, N} \tilde{\omega}\left(z_{j}, A_{j}, \mathcal{M}_{j}\right) .
$$

2) Put $\widehat{X}:=\widehat{\mathbb{X}}\left(A_{1}, \ldots, A_{N} ; \mathcal{M}_{1}, \ldots, \mathcal{M}_{N}\right)$. Then $A_{1} \times \cdots \times A_{N} \subset \widehat{X}$ and

$$
\widetilde{\omega}\left(z, A_{1} \times \cdots \times A_{N}, \widehat{X}\right)=\sum_{j=1}^{N} \widetilde{\omega}\left(z_{j}, A_{j}, \mathcal{M}_{j}\right), \quad z=\left(z_{1}, \ldots, z_{N}\right) \in \widehat{X} .
$$

Proof. Part 1) follows immediately by combining Theorem 3.1 and the work of Edigarian and Poletsky in [7].

Using Part 1), the proof of Lemma 3 in [12] still works in this context making the obviously necessary changes. 


\subsection{Three uniqueness theorems and a Two-Constant Theorem}

The following uniqueness theorems will play a key role in the sequel.

Theorem 3.8. Let $\mathcal{M}$ be a connected complex manifold, A a non locally pluripolar subset of $\mathcal{M}$, and $Z$ a complex analytic space. Let $f, g \in \mathcal{O}(\mathcal{M}, Z)$ such that $f(z)=g(z), z \in A$. Then $f \equiv g$.

Proof. Since $A$ is non locally pluripolar, there is an open subset $U \subset \mathcal{M}$ biholomorphic to an Euclidean domain ${ }^{(2)}$ such that $A \cap U$ is nonpluripolar in $U$. Consequently, we deduce from the equality $f(z)=g(z), z \in A \cap U$, that $f=g$ on $U$. Since $\mathcal{M}$ is connected, the desired conclusion of the theorem follows.

Theorem 3.9. Let $D_{j}$ be a complex manifold and $A_{j} \subset D_{j}$ a non locally pluripolar subset, $j=1, \ldots, N, N \geq 2$. Let $Y$ be a complex analytic space. Let $U_{1}$ and $U_{2}$ be two open subsets of $D_{1}$. For $k \in\{1,2\}$, let $f_{k} \in \mathcal{O}\left(\widehat{X}_{k}, Y\right)$, where

$$
\widehat{X}_{k}:=\widehat{\mathbb{X}}\left(A_{1} \cap U_{k}, A_{2}, \ldots, A_{N} ; U_{k}, D_{2}, \ldots, D_{N}\right) .
$$

Then:

1) If $f_{1}=f_{2}$ on $\left(U_{1} \cap U_{2}\right) \times\left(A_{2} \cap A_{2}^{*}\right) \times \cdots \times\left(A_{N} \cap A_{N}^{*}\right)$, then $f_{1}=f_{2}$ on $\widehat{X}_{1} \cap \widehat{X}_{2}$.

2) If $U_{1}=U_{2}$ and $f_{1}=f_{2}$ on $\left(A_{1} \cap A_{1}^{*} \cap U_{1}\right) \times\left(A_{2} \cap A_{2}^{*}\right) \times \cdots \times\left(A_{N} \cap A_{N}^{*}\right)$, then $f_{1}=f_{2}$ on $\widehat{X}_{1}$.

Proof. To prove Part 1), fix an arbitrary point $z^{0}=\left(z_{1}^{0}, \ldots, z_{N}^{0}\right) \in \widehat{X}_{1} \cap \widehat{X}_{2}$. We need to show that $f_{1}\left(z^{0}\right)=f_{2}\left(z^{0}\right)$.

For any $2 \leq j \leq N$, let $\mathcal{G}_{j}$ be the connected component which contains $z_{j}^{0}$ of the following open set

$$
\left\{z_{j} \in D_{j}: \widetilde{\omega}\left(z_{j}, A_{j}, D_{j}\right)<1-\max _{k \in\{1,2\}} \widetilde{\omega}\left(z_{1}^{0}, A \cap U_{k}, U_{k}\right)-\sum_{p=2}^{j-1} \widetilde{\omega}\left(z_{p}^{0}, A_{p}, D_{p}\right)\right\} .
$$

Observe that for $k \in\{1,2\}$ and $\left(a_{3}, \ldots, a_{N}\right) \in\left(A_{3} \cap A_{3}^{*}\right) \times \cdots \times\left(A_{N} \cap A_{N}^{*}\right)$, the mapping $z_{2} \in \mathcal{G}_{2} \mapsto f_{k}\left(z_{1}^{0}, z_{2}, a_{3}, \ldots, a_{N}\right)$ belongs to $\mathcal{O}\left(\mathcal{G}_{2}, Y\right)$. In addition, it follows from the hypothesis that

$$
f_{1}\left(z_{1}^{0}, a_{2}, \ldots, a_{N}\right)=f_{2}\left(z_{1}^{0}, a_{2}, \ldots, a_{N}\right), \quad a_{2} \in A_{2} \cap A_{2}^{*} .
$$

On the other hand, by Part 5) of Proposition 3.5, $\mathcal{G}_{2}$ contains a non locally pluripolar subset of $A_{2} \cap A_{2}^{*}$. Therefore, by Theorem 3.8,

$$
\begin{aligned}
f_{1}\left(z_{1}^{0}, z_{2}, a_{3}, \ldots, a_{N}\right) & =f_{2}\left(z_{1}^{0}, z_{2}, a_{3}, \ldots, a_{N}\right), \\
& \left(z_{2}, a_{3}, \ldots, a_{N}\right) \in \mathcal{G}_{2} \times\left(A_{3} \cap A_{3}^{*}\right) \times \cdots \times\left(A_{N} \cap A_{N}^{*}\right) .
\end{aligned}
$$

${ }^{(2)}$ An Euclidean domain is, by definition, a domain in $\mathbb{C}^{n}$. 
Hence,

$$
\begin{aligned}
f_{1}\left(z_{1}^{0}, z_{2}^{0}, a_{3}, \ldots, a_{N}\right)= & f_{2}\left(z_{1}^{0}, z_{2}^{0}, a_{3}, \ldots, a_{N}\right), \\
& \left(a_{3}, \ldots, a_{N}\right) \in\left(A_{3} \cap A_{3}^{*}\right) \times \cdots \times\left(A_{N} \cap A_{N}^{*}\right) .
\end{aligned}
$$

Repeating the argument in (3.8)-(3.9) $(N-2)$ times, we finally obtain $f_{1}\left(z^{0}\right)=$ $f_{2}\left(z^{0}\right)$. Hence, the proof of Part 1) is finished.

In virtue of Part 1), Part 2) is reduced to proving that

$$
f_{1}=f_{2} \quad \text { on } U_{1} \times\left(A_{2} \cap A_{2}^{*}\right) \times \cdots \times\left(A_{N} \cap A_{N}^{*}\right) .
$$

To do this, fix an arbitrary point $z^{0}=\left(z_{1}^{0}, a_{2}^{0} \ldots, a_{N}^{0}\right) \in U_{1} \times\left(A_{2} \cap A_{2}^{*}\right) \times \cdots \times$ $\left(A_{N} \cap A_{N}^{*}\right)$ such that $z^{0} \in \widehat{X}_{1}$. Then $\widetilde{\omega}\left(z_{1}, A_{1} \cap A_{1}^{*} \cap U_{1}, U_{1}\right)<1$. Let $\mathcal{G}$ be the connected component containing $z_{1}^{0}$ of $U_{1}$. Using Parts 1) and 3) of Proposition 3.5 and taking into account the latter estimate, we see that $A_{1} \cap A_{1}^{*} \cap \mathcal{G}$ is a non locally pluripolar set.

Next, observe that for $k \in\{1,2\}$, the mapping $z_{1} \in \mathcal{G} \mapsto f_{k}\left(z_{1}, a_{2}^{0}, \ldots, a_{N}^{0}\right)$ belongs to $\mathcal{O}\left(U_{1}, Y\right)$. Moreover, since we know from the hypothesis and the above paragraph that $f_{1}\left(\cdot, a_{2}^{0}, \ldots, a_{N}^{0}\right)=f_{2}\left(\cdot, a_{2}^{0}, \ldots, a_{N}^{0}\right)$ on the non locally pluripolar set $A_{1} \cap A_{1}^{*} \cap \mathcal{G}$, it follows from Theorem 3.8 that

$$
f_{1}\left(z_{1}, a_{2}^{0}, \ldots, a_{N}^{0}\right)=f_{2}\left(z_{1}, a_{2}^{0}, \ldots, a_{N}^{0}\right), \quad z_{1} \in \mathcal{G} .
$$

Hence, $f_{1}\left(z^{0}\right)=f_{2}\left(z^{0}\right)$, which completes the proof of Part 2).

The next result, combined with Part 2) of Theorem 3.9, establishes the uniqueness stated in Theorem A and in its intermediate versions (see Theorems 4.1, 5.1, 6.1 and Proposition 6.6 below).

Theorem 3.10. Let $D_{j}$ be a complex manifold and $A_{j} \subset D_{j}$ a non locally pluripolar subset, $j=1, \ldots, N$. Let $Z$ be a complex analytic space. One defines $X, X^{*}$ and $\widehat{X}$ as in Subsection 2.1. Let $f \in \mathcal{O}_{s}(X, Z)$ and $\hat{f} \in \mathcal{O}(\widehat{X}, Z)$ such that $\hat{f}=f$ on $X \cap X^{*}$. Then $\hat{f}=f$ on $X \cap \widehat{X}$.

Proof. Let $z^{0}=\left(z_{1}^{0}, \ldots, z_{N}^{0}\right)$ be an arbitrary point of $X \cap \widehat{X}$, and put $f_{1}:=\hat{f}$, $f_{2}:=f$. Arguing as in the proof of Theorem 3.9, we can show that $\hat{f}\left(z^{0}\right)=f\left(z^{0}\right)$. This completes the proof.

The following Two-Constant Theorem for plurisubharmonic functions will play an important role in the proof of the estimate in Theorem A.

Theorem 3.11. Let $\mathcal{M}$ be a complex manifold and A a non locally pluripolar subset of $\mathcal{M}$. Let $m, M \in \mathbb{R}$ and $u \in \mathcal{P S H}(\mathcal{M})$ such that $u(z) \leq M$ for $z \in \mathcal{M}$, and $u(z) \leq m$ for $z \in A$. Then

$$
u(z) \leq m(1-\widetilde{\omega}(z, A, \mathcal{M}))+M \cdot \widetilde{\omega}(z, A, \mathcal{M}), \quad z \in \mathcal{M} .
$$

Proof. It follows immediately from the definition of $\widetilde{\omega}(\cdot, A, \mathcal{M})$ given in Subsection 2.1 . 


\section{Part 1 of the proof of Theorem A}

The main purpose of the section is to prove Theorem A in the following special case.

Theorem 4.1. Let $D$ be a complex manifold, let $G$ be a complex manifold which is biholomorphic to an open set in $\mathbb{C}^{q}(q \in \mathbb{N})$, let $A$ be an open subset of $D$, and let $B$ be a non locally pluripolar subset of $G$. Let $Z$ be a complex analytic space possessing the Hartogs extension property. Put $X:=\mathbb{X}(A, B ; D, G)$ and $\widehat{X}:=\widehat{\mathbb{X}}(A, B ; D, G)$. Then for any mapping $f \in \mathcal{O}_{S}(X, Z)$, there is a unique mapping $\hat{f} \in \mathcal{O}(\widehat{X}, Z)$ such that $\hat{f}=f$ on $X \cap X^{*}$.

Remark 4.2. Under the above hypothesis, it can be checked that $X \cap X^{*}=(A \times$ $G) \cup\left(D \times\left(B \cap B^{*}\right)\right)$. In addition, in the proof below we assume that $G$ is a domain in $\mathbb{C}^{q}$. Clearly, in virtue of Part 3) of Proposition 3.5, it suffices to prove the theorem under this assumption.

Proof. We begin the proof with the following lemma.

Lemma 4.3. We keep the hypothesis of Theorem 4.1. For $j \in\{1,2\}$, let $\phi_{j} \in$ $\mathcal{O}(\bar{E}, D)$ be a holomorphic disc, and let $t_{j} \in E$ such that $\phi_{1}\left(t_{1}\right)=\phi_{2}\left(t_{2}\right)$ and $\frac{1}{2 \pi} \int_{0}^{2 \pi} 1_{D \backslash A}\left(\phi_{j}\left(e^{i \theta}\right)\right) d \theta<1$. Then:

1) For $j \in\{1,2\}$, the mapping $(t, w) \mapsto f(\phi(t), w)$ belongs to $\mathcal{O}_{s}\left(\mathbb{X}\left(\phi_{j}^{-1}(A) \cap\right.\right.$ $E, B ; E, G), Z)$, where $\phi_{j}^{-1}(A):=\left\{t \in \bar{E}: \phi_{j}(t) \in A\right\}$.

2) For $j \in\{1,2\}$, in virtue of Part 1), Remark 4.2 and applying Theorem 2.3, let $\hat{f}_{j}$ be the unique mapping in $\mathcal{O}\left(\widehat{\mathbb{X}}\left(\phi_{j}^{-1}(A) \cap E, B ; E, G\right), Z\right)$ such that $\hat{f}_{j}(t, w)=f\left(\phi_{j}(t), w\right),(t, w) \in \mathbb{X}\left(\phi_{j}^{-1}(A) \cap E, B \cap B^{*} ; E, G\right)$. Then

$$
\hat{f}_{1}\left(t_{1}, w\right)=\hat{f}_{2}\left(t_{2}, w\right)
$$

for all $w \in G$ such that $\left(t_{j}, w\right) \in \widehat{\mathbb{X}}\left(\phi_{j}^{-1}(A) \cap E, B ; E, G\right), j \in\{1,2\}$.

Proof of Lemma 4.3. Part 1) follows immediately from the hypothesis. Therefore, it remains to prove Part 2). To do this fix $w_{0} \in G$ such that $\left(t_{j}, w_{0}\right) \in$ $\widehat{\mathbb{X}}\left(\phi_{j}^{-1}(A) \cap E, B ; E, G\right)$ for $j \in\{1,2\}$. We need to show that $\hat{f}_{1}\left(t_{1}, w_{0}\right)=$ $\hat{f}_{2}\left(t_{2}, w_{0}\right)$. Observe that both mappings $w \in \mathcal{G} \mapsto \hat{f}_{1}\left(t_{1}, w\right)$ and $w \in \mathcal{G} \mapsto$ $\hat{f}_{2}\left(t_{2}, w\right)$ belong to $\mathcal{O}(\mathcal{G}, Z)$, where $\mathcal{G}$ is the connected component which contains $w_{0}$ of the following open set

$$
\left\{w \in G: \widetilde{\omega}(w, B, G)<1-\max _{j \in\{1,2\}} \widetilde{\omega}\left(t_{j}, \phi_{j}^{-1}(A) \cap E, E\right)\right\} .
$$


Moreover, for any $w \in B \cap B^{*}$ we have that $\widetilde{\omega}\left(w, B \cap B^{*}, G\right)=0$. Therefore, for any $j \in\{1,2\}$ one clearly gets that $\left(t_{j}, w\right) \in \mathbb{X}\left(\phi_{j}^{-1}(A) \cap E, B \cap B^{*} ; E, G\right)$. Consequently, since $\phi_{1}\left(t_{1}\right)=\phi_{2}\left(t_{2}\right)$, it follows that

$$
\hat{f}_{1}\left(t_{1}, w\right)=f\left(\phi_{1}\left(t_{1}\right), w\right)=f\left(\phi_{2}\left(t_{2}\right), w\right)=\hat{f}_{2}\left(t_{2}, w\right), \quad w \in B \cap B^{*} .
$$

On the other hand, by Part 5) of Proposition 3.5, $\mathcal{G}$ contains a non locally pluripolar subset of $B \cap B^{*}$. Therefore, by Theorem 3.8, $\hat{f}_{1}\left(t_{1}, w\right)=\hat{f}_{2}\left(t_{2}, w\right), w \in \mathcal{G}$. Hence, $\hat{f}_{1}\left(t_{1}, w_{0}\right)=\hat{f}_{2}\left(t_{2}, w_{0}\right)$, which completes the proof of the lemma.

Step 1. Construction of the extension mapping $\hat{f}$ on $\widehat{X}$.

Proof of Step 1. We define $\hat{f}$ as follows: Let $\mathcal{X}$ be the set of all pairs $(z, w) \in$ $D \times G$ with the property that there are a holomorphic disc $\phi \in \mathcal{O}(\bar{E}, D)$ and $t \in E$ such that $\phi(t)=z$ and $(t, w) \in \widehat{\mathbb{X}}\left(\phi^{-1}(A) \cap E, B ; E, G\right)$. In virtue of Theorem 2.3 , let $\hat{f}_{\phi}$ be the unique mapping in $\mathcal{O}\left(\widehat{\mathbb{X}}\left(\phi^{-1}(A) \cap E, B ; E, G\right), Z\right)$ such that

$$
\hat{f}_{\phi}(t, w)=f(\phi(t), w), \quad(t, w) \in \mathbb{X}\left(\phi^{-1}(A) \cap E, B \cap B^{*} ; E, G\right)
$$

Then the desired extension mapping $\hat{f}$ is given by

$$
\hat{f}(z, w):=\hat{f}_{\phi}(t, w) .
$$

In virtue of Part 2) of Lemma 4.3, $\hat{f}$ is well-defined on $\mathcal{X}$. We next prove that

$$
\mathcal{X}=\widehat{X}
$$

Taking (4.3) for granted, then $\hat{f}$ is well-defined on $\widehat{X}$. Moreover, it follows from formula (4.2) that for every fixed $z \in D$, the restricted mapping $\hat{f}(z, \cdot)$ is holomorphic on the open set $\{w \in G:(z, w) \in \widehat{X}\}$.

Now we return to (4.3). To prove the inclusion $\mathcal{X} \subset \widehat{X}$, let $(z, w) \in \mathcal{X}$. By the above definition of $\mathcal{X}$, one may find a holomorphic disc $\phi \in \mathcal{O}(\bar{E}, D)$, a point $t \in E$ such that $\phi(t)=z$ and $(t, w) \in \widehat{\mathbb{X}}\left(\phi^{-1}(A) \cap E, B ; E, G\right)$. Since $\widetilde{\omega}(\phi(t), A, D) \leq \widetilde{\omega}\left(t, \phi^{-1}(A) \cap E, E\right)$, it follows that

$$
\widetilde{\omega}(z, A, D)+\widetilde{\omega}(w, B, G) \leq \widetilde{\omega}\left(t, \phi^{-1}(A) \cap E, E\right)+\widetilde{\omega}(w, B, G)<1,
$$

Hence $(z, w) \in \widehat{X}$. This proves the above mentioned inclusion.

To finish the proof of (4.3), it suffices to show that $\widehat{X} \subset \mathcal{X}$. To do this, let $(z, w) \in \widehat{X}$ and fix any $\epsilon>0$ such that

$$
\epsilon<1-\widetilde{\omega}(z, A, D)-\widetilde{\omega}(w, B, G) .
$$


Applying Theorem 3.1 and Proposition 3.4, there is a holomorphic disc $\phi \in \mathcal{O}(\bar{E}, D)$ such that $\phi(0)=z$ and

$$
\frac{1}{2 \pi} \int_{0}^{2 \pi} 1_{D \backslash A}\left(\phi\left(e^{i \theta}\right)\right) d \theta<\widetilde{\omega}(z, A, D)+\epsilon .
$$

Observe that

$$
\begin{aligned}
\widetilde{\omega}\left(0, \phi^{-1}(A) \cap E, E\right)+\widetilde{\omega}(w, B, G) & \leq \frac{1}{2 \pi} \int_{0}^{2 \pi} 1_{D \backslash A}\left(\phi\left(e^{i \theta}\right)\right) d \theta+\widetilde{\omega}(w, B, G) \\
& <\widetilde{\omega}(z, A, D)+\widetilde{\omega}(w, B, G)+\epsilon<1,
\end{aligned}
$$

where the first inequality follows from an application of Lemma 3.3, the second one from (4.5), and the last one from (4.4). Hence, $(0, w) \in \widehat{\mathbb{X}}\left(\phi^{-1}(A) \cap E, B ; E, G\right)$, which implies that $(z, w) \in \mathcal{X}$. This complete the proof of (4.3). Hence Step 1 is finished.

Next, we would like to show that $\hat{f}$ satisfies the conclusion of the theorem. This will be accomplished in two steps below.

Step 2. Proof of the equality $\hat{f}=f$ on $X \cap X^{*}$.

Proof of Step 2. Let $(z, w)$ be an arbitrary point of $A \times G$. Choose the holomorphic $\operatorname{disc} \phi \in \mathcal{O}(\bar{E}, D)$ given by $\phi(t):=z, t \in \bar{E}$. Then by formula (4.2),

$$
\hat{f}(z, w)=\hat{f}_{\phi}(0, w)=f(\phi(0), w)=f(z, w), \quad w \in G .
$$

Hence, $\hat{f}=f$ on $A \times G$.

Next, let $(z, w)$ be an arbitrary point of $D \times\left(B \cap B^{*}\right)$ and let $\epsilon>0$ be such that

$$
\epsilon<1-\widetilde{\omega}(z, A, D)
$$

Applying Theorem 3.1 and Proposition 3.4, one may find a holomorphic disc $\phi \in$ $\mathcal{O}(\bar{E}, D)$ such that $\phi(0)=z$ and

$$
\frac{1}{2 \pi} \int_{0}^{2 \pi} 1_{D \backslash A}\left(\phi\left(e^{i \theta}\right)\right) d \theta<\widetilde{\omega}(z, A, D)+\epsilon .
$$

Consequently,

$\widetilde{\omega}\left(0, \phi^{-1}(A) \cap E, E\right)+\widetilde{\omega}(w, B, G) \leq \frac{1}{2 \pi} \int_{0}^{2 \pi} 1_{D \backslash A}\left(\phi\left(e^{i \theta}\right)\right) d \theta<\widetilde{\omega}(z, A, D)+\epsilon<1$, 
where the first inequality follows from an application of Lemma 3.3 and the equality $\widetilde{\omega}(w, B, G)=0$, the second one from (4.8), and the last one from (4.7). Hence, $(0, w) \in \widehat{\mathbb{X}}\left(\phi^{-1}(A) \cap E, B ; E, G\right)$. Therefore, using (4.1)-(4.2) and arguing as in (4.6), we conclude that $\hat{f}(z, w)=f(z, w)$. This proves that $\hat{f}=f$ on $D \times(B \cap$ $\left.B^{*}\right)$.

In summary, we have shown that $\hat{f}=f$ on $(A \times G) \cup\left(D \times\left(B \cap B^{*}\right)\right)$. In virtue of Remark 4.2, Step 2 is complete.

Step 3. Proof of the fact that $\hat{f} \in \mathcal{O}(\widehat{X}, Z)$.

Proof of Step 3. Fix an arbitrary point $\left(z_{0}, w_{0}\right) \in \widehat{X}$ and let $\epsilon>0$ be so small such that

$$
2 \epsilon<1-\widetilde{\omega}\left(z_{0}, A, D\right)-\widetilde{\omega}\left(w_{0}, B, G\right) .
$$

Since $\widetilde{\omega}(\cdot, B, G) \in \mathcal{P} \mathcal{S H}(G)$, one may find an open neighborhood $V$ of $w_{0}$ such that

$$
\widetilde{\omega}(w, B, D)<\widetilde{\omega}\left(w_{0}, B, G\right)+\epsilon, \quad w \in V .
$$

Let $d$ be the dimension of $D$ at the point $z_{0}$. Applying Lemma 3.2 and Proposition 3.4, we obtain an open set $T$ in $\mathbb{C}$, an open neighborhood $U$ of $z_{0}$ which is biholomorphic to the unit ball in $\mathbb{C}^{d}$, and and a family of holomorphic discs $\left(\phi_{z}\right)_{z \in U} \subset \mathcal{O}(\bar{E}, D)$ with the following properties:

$$
\begin{aligned}
& \text { the mapping }(z, t) \in U \times E \mapsto \phi_{z}(t) \text { is holomorphic; } \\
& \phi_{z}(0)=z, \quad z \in U \text {; } \\
& \phi_{z}(t) \in A, \quad t \in T \cap \bar{E}, z \in U \text {; } \\
& \frac{1}{2 \pi} \int_{0}^{2 \pi} 1_{\partial E \backslash T}\left(e^{i \theta}\right) d \theta<\widetilde{\omega}\left(z_{0}, A, D\right)+\epsilon .
\end{aligned}
$$

Consider the mapping $g: \mathbb{X}(T \cap E, U, B ; E, U, G) \longrightarrow Z$ given by

$$
g(t, z, w):=f\left(\phi_{z}(t), w\right), \quad(t, z, w) \in \mathbb{X}(T \cap E, U, B ; E, U, G) .
$$

We make the following observations:

Let $t \in T \cap E$. Then, in virtue of (4.13) we have $\phi_{z}(t) \in A$ for $z \in U$. Consequently, in virtue of (4.11), (4.15) and the hypothesis $f \in \mathcal{O}_{s}(X, Z)$, we conclude that $\left.g(t, z, \cdot)\right|_{G} \in \mathcal{O}(G, Z)\left(\operatorname{resp} .\left.g(t, \cdot, w)\right|_{U} \in \mathcal{O}(U, Z)\right)$ for any $z \in U$ (resp. $w \in B$ ). Analogously, for any $z \in U, w \in B$, we can show that $\left.g(\cdot, z, w)\right|_{E} \in \mathcal{O}(E, Z)$.

In summary, we have shown that $g \in \mathcal{O}_{s}(\mathbb{X}(T \cap E, U, B ; E, U, G), Z)$. Recall that $U$ is biholomorphic to the unit ball in $\mathbb{C}^{d}$. Consequently, we are able to apply Theorem 2.3 to $g$ in order to obtain a unique mapping

$$
\hat{g} \in \mathcal{O}(\widehat{\mathbb{X}}(T \cap E, U, B ; E, U, G), Z)
$$


such that

$$
\hat{g}(t, z, w)=g(t, z, w), \quad(t, z, w) \in \mathbb{X}\left(T \cap E, U, B \cap B^{*} ; E, U, G\right) .
$$

Observe that

$$
\begin{aligned}
\widehat{\mathbb{X}}(T \cap E, U, B ; E, U, G)= & \{(t, z, w) \in E \times U \times G: \widetilde{\omega}(t, T \cap E, E) \\
& +\widetilde{\omega}(w, B, G)<1\} .
\end{aligned}
$$

On the other hand, for any $w \in V$,

$$
\begin{aligned}
\widetilde{\omega}(0, T \cap E, E)+\widetilde{\omega}(w, B, G) & \leq \frac{1}{2 \pi} \int_{0}^{2 \pi} 1_{\partial E \backslash T}\left(e^{i \theta}\right) d \theta+\widetilde{\omega}\left(w_{0}, B, G\right)+\epsilon \\
& <\widetilde{\omega}\left(z_{0}, A, D\right)+\widetilde{\omega}\left(w_{0}, B, G\right)+2 \epsilon<1,
\end{aligned}
$$

where the first inequality follows from an application of Lemma 3.3 and (4.10), the second one from (4.14), and the last one from (4.9). Consequently,

$$
(0, z, w) \in \widehat{\mathbb{X}}(T \cap E, U, B ; E, U, G), \quad(z, w) \in U \times V .
$$

It follows from (4.2), (4.12), (4.13) and (4.17) that, for $z \in U, \hat{f}_{\phi_{z}}$ is well-defined and holomorphic on $\widehat{\mathbb{X}}(T \cap E, B ; E, G)$, and

$$
\hat{f}(z, w)=\hat{f}_{\phi_{z}}(0, w), \quad w \in V
$$

On the other hand, it follows from (4.1), (4.15) and (4.16) that

$$
\hat{f}_{\phi_{z}}(t, w)=\hat{g}(t, z, w), \quad(t, w) \in \mathbb{X}\left(T \cap E, B \cap B^{*} ; E, G\right), z \in U .
$$

Since, for fixed $z \in U$, the restricted mapping $(t, w) \mapsto \hat{g}(t, z, w)$ is holomorphic on $\widehat{\mathbb{X}}(T \cap E, B ; E, G)$, we deduce from the latter equality and the uniqueness of Theorem 2.3 that

$$
\hat{g}(t, z, w)=\hat{f}_{\phi_{z}}(t, w), \quad(t, w) \in \widehat{\mathbb{X}}\left(T \cap E, B \cap B^{*} ; E, G\right), z \in U .
$$

In particular, using (4.2), (4.18) and (4.19),

$$
\hat{g}(0, z, w)=\hat{f}_{\phi_{z}}(0, w)=\hat{f}(z, w), \quad(z, w) \in U \times V .
$$

Since we know from (4.18) that $\hat{g}$ is holomorphic on a neighborhood of $\left(0, z_{0}, w_{0}\right)$, we conclude that $\hat{f}$ is holomorphic on a neighborhood of $\left(z_{0}, w_{0}\right)$. Since $\left(z_{0}, w_{0}\right) \in$ $\widehat{X}$ is arbitrary, it follows that $\hat{f} \in \mathcal{O}(\widehat{X}, Z)$. Hence Step 3 is complete.

Combining Steps 1-3, the theorem follows. 


\section{Part 2 of the proof of Theorem A}

The main purpose of the section is to prove Theorem A in the following special case.

Theorem 5.1. Let $D, G$ be complex manifolds, and let $A \subset D, B \subset G$ be open subsets. Let $Z$ be a complex analytic space possessing the Hartogs extension property. Put $X:=\mathbb{X}(A, B ; D, G)$ and $\widehat{X}:=\widehat{\mathbb{X}}(A, B ; D, G)$. Then for any mapping $f \in \mathcal{O}_{s}(X, Z)$, there is a unique mapping $\hat{f} \in \mathcal{O}(\widehat{X}, Z)$ such that $\hat{f}=f$ on $X$.

Remark 5.2. Using Part 1) of Proposition 3.5 it can be checked that, under the above hypothesis, $X^{*}=X$.

Proof. Let us start with the following lemma.

Lemma 5.3. We keep the hypothesis of Theorem 5.1. For $j \in\{1,2\}$, let $\psi_{j} \in$ $\mathcal{O}(\bar{E}, G)$ be a holomorphic disc and let $\tau_{j} \in E$ such that $\psi_{1}\left(\tau_{1}\right)=\psi_{2}\left(\tau_{2}\right)$ and $\frac{1}{2 \pi} \int_{0}^{2 \pi} 1_{G \backslash B}\left(\psi_{j}\left(e^{i \theta}\right)\right) d \theta<1$. Then:

1) For $j \in\{1,2\}$, the mapping $(z, \tau) \mapsto f\left(z, \psi_{j}(\tau)\right)$ belongs to

$$
\mathcal{O}_{s}\left(\mathbb{X}\left(A, \psi_{j}^{-1}(B) \cap E ; D, E\right), Z\right),
$$

where $\psi_{j}^{-1}(B):=\left\{\tau \in \bar{E}: \psi_{j}(\tau) \in B\right\}$.

2) For $j \in\{1,2\}$, in virtue of Part 1), Remark 5.2 and applying Theorem 4.1, let $\tilde{f}_{j}$ be the unique mapping in $\mathcal{O}\left(\widehat{\mathbb{X}}\left(A, \psi_{j}^{-1}(B) \cap E ; D, E\right), Z\right)$ such that $\tilde{f}_{j}(z, \tau)=f\left(z, \psi_{j}(\tau)\right),(z, \tau) \in \mathbb{X}\left(A, \psi_{j}^{-1}(B) \cap E ; D, E\right)$. Then

$$
\tilde{f}_{1}\left(z, \tau_{1}\right)=\tilde{f}_{2}\left(z, \tau_{2}\right)
$$

for all $z \in D$ such that $\left(z, \tau_{j}\right) \in \widehat{\mathbb{X}}\left(A, \psi_{j}^{-1}(B) \cap E ; D, E\right)$, for $j \in\{1,2\}$.

Proof of Lemma 5.3. It follows along the same lines as those of Lemma 4.3.

Now we return to Theorem 5.1. First we define a mapping $\hat{g}: \widehat{X} \longrightarrow Z$ as follows: Let $\mathcal{X}$ be the set of all pairs $(z, w) \in D \times G$ with the property that there are a holomorphic disc $\psi \in \mathcal{O}(\bar{E}, G)$ and $\tau \in E$ such that $\psi(\tau)=w$ and $(z, \tau) \in \widehat{\mathbb{X}}\left(A, \psi^{-1}(B) \cap E ; D, E\right)$. In virtue of Lemma 5.3, let $\tilde{f}_{\psi}$ be the unique mapping in $\mathcal{O}\left(\widehat{\mathbb{X}}\left(A, \psi^{-1}(B) \cap E ; D, E\right), Z\right)$ such that

$$
\tilde{f}_{\psi}(z, \tau)=f(z, \psi(\tau)), \quad(z, \tau) \in \mathbb{X}\left(A, \psi^{-1}(B) \cap E ; D, E\right) .
$$


Then we define

$$
\hat{g}(z, w):=\tilde{f}_{\psi}(z, \tau)
$$

In virtue of Part 2) of Lemma 5.3, $\hat{g}$ is well-defined on $\mathcal{X}$. Moreover, arguing as in Step 1 in the proof of Theorem 4.1, we can show that $\mathcal{X}=\widehat{X}$. Consequently, $\hat{g}$ is well-defined on $\widehat{X}$. Moreover, arguing as in Step 2 in the proof of Theorem 4.1, it follows from (5.1)-(5.2) and Remark 5.2 that for every fixed $w \in G$, the restricted mapping $\hat{g}(\cdot, w)$ is holomorphic on the open set $\{z \in D:(z, w) \in \widehat{X}\}$ and that $\hat{g}=f$ on $X$.

We define the desired mapping $\hat{f}$ on $\widehat{X}$ as follows: Let $(z, w) \in \widehat{X}$, let $\phi \in$ $\mathcal{O}(\bar{E}, D)$ be a holomorphic disc and $t \in E$ such that $\phi(t)=z$ and $(t, w) \in \widehat{\mathbb{X}}$ $\left(\phi^{-1}(A) \cap E, B ; E, G\right)$. In virtue of Lemma 5.3 and replacing the role of $B$ (resp. $D$ ) by that of $A$ (resp. $G$ ) therein, let $\hat{f}_{\phi}$ be the unique mapping in

$$
\mathcal{O}\left(\widehat{\mathbb{X}}\left(\phi^{-1}(A) \cap E, B ; E, G\right), Z\right)
$$

such that

$$
\hat{f}_{\phi}(t, w)=f(\phi(t), w), \quad(t, w) \in \mathbb{X}\left(\phi^{-1}(A) \cap E, B ; E, G\right) .
$$

Then we put

$$
\hat{f}(z, w):=\hat{f}_{\phi}(t, w)
$$

Arguing as in the previous paragraph, we conclude that $\hat{f}$ is well-defined on $\widehat{X}$. Moreover, it follows from (5.3)-(5.4) and Remark 5.2 that for every fixed $z \in D$, the restricted mapping $\hat{f}(z, \cdot)$ is holomorphic on the open set $\{w \in G:(z, w) \in \widehat{X}\}$ and that $\hat{f}=f$ on $X$.

The proof of the theorem will be complete if we can show that

$$
\hat{f}=\hat{g} \text {. }
$$

Indeed, taking (5.5) for granted, then for any $\left(z_{0}, w_{0}\right) \in \widehat{X}$, we may find an open neighborhood $U \times V$ of $\left(z_{0}, w_{0}\right)$ such that $U \times V \subset \widehat{X}$ and $U$ (resp. $V$ ) is biholomorphic to an Euclidean ball. Using (5.5) and the above-mentioned property of $\hat{f}$ and $\hat{g}$, we see that $\hat{f}(=\hat{g}) \in \mathcal{O}_{s}(\mathbb{X}(U, V ; U, V), Z)$. Consequently, applying Theorem 2.3 to $\hat{f}$, it follows that $\hat{f} \in \mathcal{O}(U \times V, Z)$. Hence, $\hat{f} \in \mathcal{O}(\widehat{X}, Z)$, and the proof of the theorem is finished.

To prove $(5.5)$, fix an arbitrary point $\left(z_{0}, w_{0}\right) \in \widehat{X}$. Fix any $\epsilon>0$ such that

$$
3 \epsilon<1-\widetilde{\omega}\left(z_{0}, A, D\right)-\widetilde{\omega}\left(w_{0}, B, G\right)
$$


Applying Theorem 3.1 and Proposition 3.4, there is a holomorphic $\operatorname{disc} \phi \in \mathcal{O}(\bar{E}, D)$ (resp. $\psi \in \mathcal{O}(\bar{E}, G))$ such that $\phi(0)=z_{0}\left(\right.$ resp. $\left.\psi(0)=w_{0}\right)$ and

$$
\begin{aligned}
& \frac{1}{2 \pi} \int_{0}^{2 \pi} 1_{D \backslash A}\left(\phi\left(e^{i \theta}\right)\right) d \theta<\widetilde{\omega}\left(z_{0}, A, D\right)+\epsilon, \\
& \frac{1}{2 \pi} \int_{0}^{2 \pi} 1_{G \backslash B}\left(\psi\left(e^{i \theta}\right)\right) d \theta<\widetilde{\omega}\left(w_{0}, B, G\right)+\epsilon .
\end{aligned}
$$

Using this and estimate (5.6), and arguing as in Step 1 of Theorem 4.1, we see that $(0,0) \in \widehat{\mathbb{X}}\left(\phi^{-1}(A) \cap E, \psi^{-1}(B) \cap E ; E, E\right)$. Moreover, since $f \in \mathcal{O}_{s}(X, Z)$, the mapping $h$ given by

$$
h(t, \tau):=f(\phi(t), \psi(\tau)), \quad(t, \tau) \in \mathbb{X}\left(\phi^{-1}(A) \cap E, \psi^{-1}(B) \cap E ; E, E\right),
$$

belongs to $\mathcal{O}_{s}\left(\mathbb{X}\left(\phi^{-1}(A) \cap E, \psi^{-1}(B) \cap E ; E, E\right), Z\right)$. Moreover, in virtue of (5.1) and (5.3),

$$
\begin{gathered}
\hat{f}_{\phi}(t, \psi(\tau))=f(\phi(t), \psi(\tau))=\tilde{f}_{\psi}(\phi(t), \tau), \\
(t, \tau) \in \mathbb{X}\left(\phi^{-1}(A) \cap E, \psi^{-1}(B) \cap E ; E, E\right) .
\end{gathered}
$$

By Theorem 2.3, let $\hat{h} \in \mathcal{O}\left(\widehat{\mathbb{X}}\left(\phi^{-1}(A) \cap E, \psi^{-1}(B) \cap E ; E, E\right), Z\right)$ be the unique mapping such that

$$
\hat{h}(t, \tau)=h(t, \tau)=f(\phi(t), \psi(\tau)), \quad(t, \tau) \in \mathbb{X}\left(\phi^{-1}(A) \cap E, \psi^{-1}(B) \cap E ; E, E\right) .
$$

Then in virtue of (5.7) we clearly have that

$$
\hat{f}_{\phi}(t, \psi(\tau))=\hat{h}(t, \tau)=\tilde{f}_{\psi}(\phi(t), \tau),(t, \tau) \in \widehat{\mathbb{X}}\left(\phi^{-1}(A) \cap E, \psi^{-1}(B) \cap E ; E, E\right) .
$$

Therefore,

$$
\hat{f}_{\phi}\left(0, w_{0}\right)=\hat{h}(0,0)=\tilde{f}_{\psi}\left(z_{0}, 0\right),
$$

which, in turn, implies that

$$
\hat{f}\left(z_{0}, w_{0}\right)=\hat{f}_{\phi}\left(0, w_{0}\right)=\tilde{f}_{\psi}\left(z_{0}, 0\right)=\hat{g}\left(z_{0}, w_{0}\right) .
$$

Hence, the proof of identity (5.5) is complete. This finishes the proof of the theorem. 


\section{Part 3 of the proof: Theorem A for the case $N=2$}

The main purpose of the section is to prove Theorem A for the case $N=2$.

Theorem 6.1. Let $D, G$ be complex manifolds, and let $A \subset D, B \subset G$ be non locally pluripolar subsets. Let $Z$ be a complex analytic space possessing the Hartogs extension property. Put $X:=\mathbb{X}(A, B ; D, G)$ and $\widehat{X}:=\widehat{\mathbb{X}}(A, B ; D, G)$. Then for any mapping $f \in \mathcal{O}_{S}(X, Z)$, there is a unique mapping $\hat{f} \in \mathcal{O}(\widehat{X}, Z)$ such that $\hat{f}=f$ on $X \cap X^{*}$.

For the proof we need to develop some preparatory results.

For any $a \in A^{*}$ (resp. $b \in B^{*}$ ), fix an open neighborhood $U_{a}$ of $a$ (resp. $V_{b}$ of $b$ ) such that $U_{a}$ (resp. $\left.V_{b}\right)$ is biholomorphic to a domain in $\mathbb{C}^{d_{a}}$ (resp. in $\mathbb{C}^{d_{b}}$ ), where $d_{a}$ (resp. $d_{b}$ ) is the dimension of $D\left(\right.$ resp. $G$ ) at $a\left(\right.$ resp. $b$ ). For any $0<\delta \leq \frac{1}{2}$, define

$$
\begin{aligned}
U_{a, \delta} & :=\left\{z \in U_{a}: \widetilde{\omega}\left(z, A \cap U_{a}, U_{a}\right)<\delta\right\}, \quad a \in A \cap A^{*}, \\
V_{b, \delta} & :=\left\{w \in V_{b}: \widetilde{\omega}\left(w, B \cap V_{b}, V_{b}\right)<\delta\right\}, \quad b \in B \cap B^{*}, \\
A_{\delta} & :=\bigcup_{a \in A \cap A^{*}} U_{a, \delta}, \quad B_{\delta}:=\bigcup_{b \in B \cap B^{*}} V_{b, \delta}, \\
D_{\delta} & :=\{z \in D: \widetilde{\omega}(z, A, D)<1-\delta\}, \quad G_{\delta}:=\{w \in G: \widetilde{\omega}(w, B, G)<1-\delta\} .
\end{aligned}
$$

Lemma 6.2. We keep the above notation. Then

$$
\begin{aligned}
A \cap A^{*} & \subset A_{\delta} \subset D_{1-\delta} \subset D_{\delta}, \\
\widetilde{\omega}(z, A, D)-\delta & \leq \widetilde{\omega}\left(z, A_{\delta}, D\right) \leq \widetilde{\omega}(z, A, D), \quad z \in D .
\end{aligned}
$$

Proof of Lemma 6.2. Using (6.1) and the definition of local pluriregularity, we see that $a \in U_{a, \delta}$ for $a \in A \cap A^{*}$. Consequently, the first inclusion in (6.2) follows. Since $0<\delta \leq \frac{1}{2}$, the third inclusion in (6.2) is clear. To prove the second inclusion in (6.2), let $z$ be an arbitrary point of $A_{\delta}$. Then there is an $a \in A \cap A^{*}$ such that $z \in U_{a, \delta}$. Applying Part 2) of Proposition 3.5 and taking into account the inequality $0<\delta \leq \frac{1}{2}$, we obtain

$$
\widetilde{\omega}(z, A, D) \leq \widetilde{\omega}\left(z, A \cap U_{a}, U_{a}\right)<\delta .
$$

Hence, $z \in D_{1-\delta}$, which in turn implies that $A_{\delta} \subset D_{1-\delta}$. Hence, all assertions in (6.2) are proved.

Next, using the first inclusion in (6.2) and applying Parts 1) and 2) of Proposition 3.5 , we get

$$
\widetilde{\omega}\left(z, A_{\delta}, D\right) \leq \widetilde{\omega}\left(z, A \cap A^{*}, D\right)=\widetilde{\omega}(z, A, D), \quad z \in D,
$$

which proves the second estimate in (6.3). 
To complete the proof of (6.3), let $a \in A \cap A^{*}$ and $0<\delta \leq \frac{1}{2}$. We deduce from (6.4) that $\widetilde{\omega}(z, A, D)-\delta \leq 0$ for $z \in U_{a, \delta}$. Hence, by (6.1),

$$
\widetilde{\omega}(z, A, D)-\delta \leq 0, \quad z \in A_{\delta} .
$$

On the other hand, $\widetilde{\omega}(z, A, D)-\delta<1, z \in D$. Consequently, the first estimate in (6.3) follows. Hence, the proof of the lemma is finished.

We also need the following

Definition 6.3. Let $\mathcal{M}$ be a complex manifold and $Y$ a complex space. Let $\left(U_{j}\right)_{j \in J}$ be a family of open subsets of $\mathcal{M}$, and $\left(f_{j}\right)_{j \in J}$ a family of mappings such that $f_{j} \in \mathcal{O}\left(U_{j}, Y\right)$. We say that the family $\left(f_{j}\right)_{j \in J}$ is "collective" if, for any $j, k \in J$, $f_{j}=f_{k}$ on $U_{j} \cap U_{k}$. The unique holomorphic mapping $f: \bigcup_{j \in J} U_{j} \longrightarrow Y$, defined by $f:=f_{j}$ on $U_{j}, j \in J$, is called the "collected mapping" of $\left(f_{j}\right)_{j \in J}$.

Lemma 6.4. We keep the the hypothesis of Theorem 6.1 and the above notation. Suppose, in addition, that for every a $\in A \cap A^{*}$, there is a (unique) mapping $\hat{f}_{a} \in \mathcal{O}\left(\widehat{\mathbb{X}}\left(A \cap U_{a}, B ; U_{a}, G\right), Z\right)$ such that

$$
\hat{f}_{a}(z, w)=f(z, w),(z, w) \in \mathbb{X}\left(A \cap A^{*} \cap U_{a}, B \cap B^{*} ; U_{a}, G\right) .
$$

Then the family $\left(\left.\hat{f}_{a}\right|_{U_{a, \delta} \times G_{\delta}}\right)_{a \in A \cap A^{*}}$ is collective.

Proof of Lemma 6.4. Let $a_{1}, a_{2}$ be arbitrary elements of $A \cap A^{*}$. By (6.5), we have that

$$
\hat{f}_{a_{1}}(z, w)=f(z, w)=\hat{f}_{a_{2}}(z, w), \quad(z, w) \in\left(U_{a_{1}} \cap U_{a_{2}}\right) \times\left(B \cap B^{*}\right) .
$$

Consequently, in virtue of Part 1) of Theorem 3.9,

$$
\hat{f}_{a_{1}}(z, w)=\hat{f}_{a_{2}}(z, w), \quad(z, w) \in \widehat{\mathbb{X}}\left(A \cap U_{a_{1}}, B ; U_{a_{1}}, G\right) \cap \widehat{\mathbb{X}}\left(A \cap U_{a_{2}}, B ; U_{a_{2}}, G\right) .
$$

This, combined with the definition of $U_{a, \delta}$ and $G_{\delta}$ given in (6.1), the fact that $0<\delta \leq \frac{1}{2}$, and Definition 6.3, implies the desired conclusion.

Lemma 6.5. Let $\mathcal{D}$ and $\mathcal{G}$ be two complex manifolds. Let $\left(\mathcal{A}_{\delta}\right)_{0<\delta<\frac{1}{2}}$ (resp. $\left(\mathcal{B}_{\delta}\right)_{0<\delta<\frac{1}{2}}$ ) be a family of non locally pluripolar subsets of $\mathcal{D}$ (resp. $\mathcal{G}$ ), and $\left(\mathcal{D}_{\delta}\right)_{0<\delta<\frac{1}{2}}\left(\right.$ resp. $\left.\left(\mathcal{G}_{\delta}\right)_{0<\delta<\frac{1}{2}}\right)$ a family of open subsets of $\mathcal{D}($ resp. $\mathcal{G})$ with the following properties:

(i) $\mathcal{A}_{\delta_{1}} \subset \mathcal{A}_{\delta_{2}} \subset \mathcal{D}_{\delta_{2}} \subset \mathcal{D}_{\delta_{1}}$ and $\mathcal{B}_{\delta_{1}} \subset \mathcal{B}_{\delta_{2}} \subset \mathcal{G}_{\delta_{2}} \subset \mathcal{G}_{\delta_{1}}$ for $0<\delta_{1} \leq \delta_{2}<\frac{1}{2}$. 
(ii) There is a family of holomorphic mappings $\left(\hat{f}_{\delta}\right)_{0<\delta<\frac{1}{2}}$ such that

$$
\hat{f_{\delta}} \in \mathcal{O}\left(\widehat{\mathbb{X}}\left(\mathcal{A}_{\delta}, \mathcal{B}_{\delta} ; \mathcal{D}_{\delta}, \mathcal{G}_{\delta}\right), Z\right)
$$

and for $0<\delta_{1}<\delta_{2}<\frac{1}{2}$,

$$
\hat{f}_{\delta_{1}}(z, w)=\hat{f}_{\delta_{2}}(z, w), \quad(z, w) \in \mathcal{A}_{\delta_{1}} \times \mathcal{B}_{\delta_{1}} .
$$

(iii) There are an open subset $U$ (resp. V) of $\mathcal{D}($ resp. $\mathcal{G})$ and a number $0<\delta_{0}<\frac{1}{2}$ such that $\widetilde{\omega}\left(z, \mathcal{A}_{\delta}, \mathcal{D}_{\delta_{0}}\right)+\widetilde{\omega}\left(w, \mathcal{B}_{\delta}, \mathcal{G}_{\delta_{0}}\right)<1$ for all $(z, w) \in U \times V$ and $0<\delta<\delta_{0}$.

Then $\hat{f}_{\delta}(z, w)=\hat{f}_{\delta_{0}}(z, w)$ for all $(z, w) \in U \times V$ and $0<\delta<\delta_{0}$.

Proof of Lemma 6.5. Fix $\delta$ such that $0<\delta<\delta_{0}$. By (iii), we have that

$$
U \times V \subset H:=\widehat{\mathbb{X}}\left(\mathcal{A}_{\delta}, \mathcal{B}_{\delta} ; \mathcal{D}_{\delta_{0}}, \mathcal{G}_{\delta_{0}}\right)
$$

On the other hand, using (i) and Part 2) of Proposition 3.5, we see that

$$
H \subset \widehat{\mathbb{X}}\left(\mathcal{A}_{\delta}, \mathcal{B}_{\delta} ; \mathcal{D}_{\delta}, \mathcal{G}_{\delta}\right) \cap \widehat{\mathbb{X}}\left(\mathcal{A}_{\delta_{0}}, \mathcal{B}_{\delta_{0}} ; \mathcal{D}_{\delta_{0}}, \mathcal{G}_{\delta_{0}}\right)
$$

Using this and (ii), we are able to apply Part 2) of Theorem 3.9 to $\left.\hat{f}_{\delta}\right|_{H}$ and $\left.\hat{f}_{\delta_{0}}\right|_{H}$. Consequently, $\hat{f}_{\delta}=\hat{f}_{\delta_{0}}$ on $H$. This, combined with (6.6), completes the proof of the lemma.

Now we are able to to prove Theorem 6.1 in the following special case.

Proposition 6.6. We keep the hypothesis of Theorem 6.1. Suppose in addition that $G$ is biholomorphic to a domain in $\mathbb{C}^{q}(q \in \mathbb{N})$. Then the conclusion of Theorem 6.1 holds.

Proof of Proposition 6.6. For each $a \in A \cap A^{*}$, let $f_{a}:=\left.f\right|_{\mathbb{X}\left(A \cap U_{a}, B ; U_{a}, G\right)}$. Since $f \in \mathcal{O}_{s}(X, Z)$, we deduce that $f_{a} \in \mathcal{O}_{s}\left(\mathbb{X}\left(A \cap U_{a}, B ; U_{a}, G\right), Z\right)$. Recall that $U_{a}$ (resp. $G$ ) is biholomorphic to a domain in $\mathbb{C}^{d_{a}}$ (resp. in $\mathbb{C}^{q}$ ). Consequently, applying Theorem 2.3 to $f_{a}$ yields that there is a unique mapping $\hat{f}_{a} \in$ $\mathcal{O}\left(\widehat{\mathbb{X}}\left(A \cap U_{a}, B ; U_{a}, G\right), Z\right)$ such that

$$
\hat{f}_{a}(z, w)=f_{a}(z, w)=f(z, w),(z, w) \in \mathbb{X}\left(A \cap A^{*} \cap U_{a}, B \cap B^{*} ; U_{a}, G\right) .
$$

Let $0<\delta \leq \frac{1}{2}$. In virtue of (6.7), we are able to apply Lemma 6.4 to the family $\left(\left.\hat{f}_{a}\right|_{U_{a, \delta} \times G_{\delta}}\right)_{a \in A \cap A^{*}}$. Let

$$
\tilde{\tilde{f}}_{\delta} \in \mathcal{O}\left(A_{\delta} \times G_{\delta}, Z\right)
$$


denote the collected mapping of this family. In virtue of (6.7)-(6.8), we are able to define a new mapping $\tilde{f}_{\delta}$ on $\mathbb{X}\left(A_{\delta}, B \cap B^{*} ; D, G_{\delta}\right)$ as follows

$$
\tilde{f}_{\delta}:= \begin{cases}\tilde{f}_{\delta}, & \text { on } A_{\delta} \times G_{\delta}, \\ f, & \text { on } D \times\left(B \cap B^{*}\right) .\end{cases}
$$

Using this and (6.7)-(6.8), we see that $\tilde{f}_{\delta} \in \mathcal{O}_{s}\left(\mathbb{X}\left(A_{\delta}, B \cap B^{*} ; D, G_{\delta}\right), Z\right)$, and

$$
\tilde{f}_{\delta}=f \quad \text { on } \mathbb{X}\left(A \cap A^{*}, B \cap B^{*} ; D, G_{\delta}\right) .
$$

Since $A_{\delta}$ is open and $G_{\delta}$ is biholomorphic to an open set in $\mathbb{C}^{q}$, we are able to apply Theorem 4.1 to $\tilde{f}_{\delta}$ in order to obtain a mapping $\hat{f}_{\delta} \in \mathcal{O}\left(\widehat{\mathbb{X}}\left(A_{\delta}, B \cap B^{*} ; D, G_{\delta}\right), Z\right)$ such that

$$
\hat{f}_{\delta}=\tilde{f}_{\delta} \quad \text { on } \mathbb{X}\left(A_{\delta}, B \cap B^{*} ; D, G_{\delta}\right) .
$$

We are now in a position to define the desired extension mapping $\hat{f}$. Indeed, one glues $\left(\hat{f}_{\delta}\right)_{0<\delta \leq \frac{1}{2}}$ together to obtain $\hat{f}$ in the following way

$$
\hat{f}:=\lim _{\delta \rightarrow 0} \hat{f}_{\delta} \quad \text { on } \widehat{X} .
$$

One needs to check that the limit (6.11) exists and possesses all the required properties. In virtue of (6.9)-(6.11), the fact that $G_{\delta} \nearrow G$ as $\delta \searrow 0$ (by (6.1)) and Lemma 6.5 , the proof will be complete if we can show that for every $\left(z_{0}, w_{0}\right) \in \widehat{X}$, there are an open neighborhood $U \times V$ of $\left(z_{0}, w_{0}\right)$ and $\delta_{0}>0$ such that the hypothesis of Lemma 6.5 is fulfilled with

$$
\mathcal{D}:=D, \mathcal{G}:=G, \quad \mathcal{A}_{\delta}:=A_{\delta}, \quad \mathcal{B}_{\delta}:=B \cap B^{*}, \quad \mathcal{D}_{\delta}:=D, \quad \mathcal{G}_{\delta}:=G_{\delta}, \quad 0<\delta<\frac{1}{2} .
$$

To this end let

$$
\delta_{0}:=\frac{1-\widetilde{\omega}\left(z_{0}, A, D\right)-\widetilde{\omega}\left(w_{0}, B, G\right)}{2},
$$

and let $U \times V$ be an open neighborhood of $\left(z_{0}, w_{0}\right)$ such that

$$
\widetilde{\omega}(z, A, D)+\widetilde{\omega}(w, B, G)<\widetilde{\omega}\left(z_{0}, A, D\right)+\widetilde{\omega}\left(w_{0}, B, G\right)+\delta_{0} .
$$

Then for $0<\delta<\delta_{0}$ and for $(z, w) \in U \times V$, using (6.12)-(6.13) and invoking Part 4) of Proposition 3.5, we see that

$$
\begin{aligned}
\widetilde{\omega}\left(z, A_{\delta}, D\right)+\widetilde{\omega}\left(w, B \cap B^{*}, G_{\delta_{0}}\right) & \leq \widetilde{\omega}(z, A, D)+\frac{\widetilde{\omega}(w, B, G)}{1-\delta_{0}} \\
& \leq \frac{\widetilde{\omega}(z, A, D)+\widetilde{\omega}(w, B, G)}{1-\delta_{0}}<1 .
\end{aligned}
$$


This proves the above assertion. Hence, the proof of the proposition is finished.

We now arrive at

Proof of Theorem 6.1. For each $a \in A \cap A^{*}$, let $f_{a}:=\left.f\right|_{\mathbb{X}\left(A \cap U_{a}, B ; U_{a}, G\right)}$. Since $f \in \mathcal{O}_{s}(X, Z)$, we deduce that $f_{a} \in \mathcal{O}_{s}\left(\mathbb{X}\left(A \cap U_{a}, B ; U_{a}, G\right), Z\right)$. Since $U_{a}$ is biholomorphic to a domain in $\mathbb{C}^{d_{a}}$, we are able to apply Proposition 6.6 to $f_{a}$. Consequently, there is a unique mapping $\hat{f}_{a} \in \mathcal{O}\left(\widehat{\mathbb{X}}\left(A \cap U_{a}, B ; U_{a}, G\right), Z\right)$ such that

$$
\hat{f}_{a}(z, w)=f(z, w), \quad(z, w) \in \mathbb{X}\left(A \cap A^{*} \cap U_{a}, B \cap B^{*} ; U_{a}, G\right) .
$$

Let $0<\delta \leq \frac{1}{2}$. In virtue of (6.15), we may apply Lemma 6.4. Consequently, we can collect the family $\left(\left.\hat{f}_{a}\right|_{U_{a, \delta} \times G_{\delta}}\right)_{a \in A \cap A^{*}}$ in order to obtain the collected mapping $\tilde{f}_{\delta}^{A} \in \mathcal{O}\left(A_{\delta} \times G_{\delta}, Z\right)$.

Similarly, for each $b \in B \cap B^{*}$, one obtains a unique mapping

$$
\hat{f}_{b} \in \mathcal{O}\left(\widehat{\mathbb{X}}\left(A, B \cap V_{b} ; D, V_{b}\right), Z\right)
$$

such that

$$
\hat{f}_{b}(z, w)=f(z, w), \quad(z, w) \in \mathbb{X}\left(A \cap A^{*}, B \cap B^{*} \cap V_{b} ; D, V_{b}\right) .
$$

Moreover, one can collect the family $\left(\left.\hat{f}_{b}\right|_{D_{\delta} \times V_{b, \delta}}\right)_{b \in B \cap B^{*}}$ in order to obtain the collected mapping $\tilde{f}_{\delta}^{B} \in \mathcal{O}\left(D_{\delta} \times B_{\delta}, Z\right)$.

Next, we prove that

$$
\tilde{f}_{\delta}^{A}=\tilde{f}_{\delta}^{B} \quad \text { on } A_{\delta} \times B_{\delta}
$$

Indeed, in virtue of (6.15)-(6.16) it suffices to show that for any $a \in A \cap A^{*}$ and $b \in B \cap B^{*}$ and any $0<\delta \leq \frac{1}{2}$,

$$
\hat{f}_{a}(z, w)=\hat{f}_{b}(z, w), \quad(z, w) \in U_{a, \delta} \times V_{b, \delta} .
$$

Observe that in virtue of (6.15)-(6.16) one has that

$$
\hat{f}_{a}(z, w)=\hat{f}_{b}(z, w)=f(z, w), \quad(z, w) \in \mathbb{X}\left(A \cap A^{*} \cap U_{a}, B \cap B^{*} \cap V_{b} ; U_{a}, V_{b}\right) .
$$

Recall that $U_{a}$ (resp. $V_{b}$ ) is biholomorphic to a domain in $\mathbb{C}^{d_{a}}\left(\right.$ resp. $\left.\mathbb{C}^{d_{b}}\right)$. Consequently, applying the uniqueness of Theorem 2.3 yields that

$$
\hat{f}_{a}(z, w)=\hat{f}_{b}(z, w), \quad(z, w) \in \widehat{\mathbb{X}}\left(A \cap U_{a}, B \cap V_{b} ; U_{a}, V_{b}\right) .
$$

Hence, the proof of (6.18) and then the proof of (6.17) are finished. 
In virtue of (6.17), we are able to define a new mapping $\tilde{f}_{\delta}: \mathbb{X}\left(A_{\delta}, B_{\delta} ; D_{\delta}, G_{\delta}\right) \longrightarrow$ $Z$ as follows

$$
\tilde{f}_{\delta}:= \begin{cases}\tilde{f}_{\delta}^{A}, & \text { on } A_{\delta} \times G_{\delta}, \\ \tilde{f}_{\delta}^{B}, & \text { on } D_{\delta} \times B_{\delta} .\end{cases}
$$

Using formula (6.19) it can be readily checked that $\tilde{f}_{\delta} \in \mathcal{O}_{s}\left(\mathbb{X}\left(A_{\delta}, B_{\delta} ; D_{\delta}, G_{\delta}\right), Z\right)$. Since we know from (6.2) that $A_{\delta}$ (resp. $B_{\delta}$ ) is an open subset of $D_{\delta}$ (resp. $G_{\delta}$ ), we are able to apply Theorem 5.1 to $\tilde{f}_{\delta}$ for every $0<\delta \leq \frac{1}{2}$. Consequently, one obtains a unique mapping $\hat{f}_{\delta} \in \mathcal{O}\left(\widehat{\mathbb{X}}\left(A_{\delta}, B_{\delta} ; D_{\delta}, G_{\delta}\right), Z\right)$ such that

$$
\hat{f}_{\delta}=\tilde{f}_{\delta} \quad \text { on } \mathbb{X}\left(A_{\delta}, B_{\delta} ; D_{\delta}, G_{\delta}\right) .
$$

It follows from (6.15)-(6.16) and (6.19)-(6.20) that

$$
\hat{f}_{\delta}=f \quad \text { on } \mathbb{X}\left(A \cap A^{*}, B \cap B^{*} ; D_{\delta}, G_{\delta}\right) .
$$

In addition, for any $0<\delta \leq \delta_{0} \leq \frac{1}{2}$, and any $(z, w) \in A_{\delta} \times B_{\delta}$, there is an $a \in A \cap A^{*}$ such that $z \in U_{a, \delta_{0}}$. Therefore, it follows from the construction of $\tilde{f}_{\delta}^{A}$, (6.19) and (6.20) that

$$
\hat{f}_{\delta}(z, w)=\hat{f}_{a}(z, w)=\hat{f}_{\delta_{0}}(z, w) .
$$

This proves that

$$
\hat{f}_{\delta}=\hat{f}_{\delta_{0}} \quad \text { on } A_{\delta} \times B_{\delta}, 0<\delta \leq \delta_{0} \leq \frac{1}{2} .
$$

We are now in a position to define the desired extension function $\hat{f}$.

$$
\hat{f}:=\lim _{\delta \rightarrow 0} \hat{f}_{\delta} \quad \text { on } \widehat{\mathbb{X}} \text {. }
$$

To prove that $\hat{f}$ satisfies the desired conclusion of the theorem one proceeds as in the end of the proof of Proposition 6.6. In virtue of (6.21)-(6.22) and Lemma 6.5, the proof will be complete if we can verify that for every $\left(z_{0}, w_{0}\right) \in \widehat{X}$, there are an open neighborhood $U \times V$ of $\left(z_{0}, w_{0}\right)$ and $\delta_{0}>0$ such that the hypothesis of Lemma 6.5 is fulfilled with

$$
\mathcal{D}:=D, \mathcal{G}:=G, \mathcal{A}_{\delta}:=A_{\delta}, \mathcal{B}_{\delta}:=B_{\delta}, \mathcal{D}_{\delta}:=D_{\delta}, \mathcal{G}_{\delta}:=G_{\delta}, \quad 0<\delta<\frac{1}{2} .
$$

Since the verification follows along almost the same lines as (6.12)-(6.14), it is therefore left to the interested reader.

Hence, the proof of the theorem is finished. 


\section{Part 4: Completion of the proof of Theorem A}

In this section we prove Theorem A for every $N \geq 3$. We divide the proof into two parts.

\subsection{Proof of the existence and uniqueness of $\hat{f}$}

We proceed by induction (I) on $N \geq 3$. Suppose the theorem is true for $N-1 \geq 2$. We have to discuss the case of an $N$-fold cross $X:=\mathbb{X}\left(A_{1}, \ldots, A_{N} ; D_{1}, \ldots, D_{N}\right)$, where $D_{1}, \ldots, D_{N}$ are complex manifolds and $A_{1} \subset D_{1}, \ldots, A_{N} \subset D_{N}$ are non locally pluripolar subsets $(1 \leq j \leq N)$. Let $f \in \mathcal{O}_{S}(X, Z)$. Observe that the uniqueness of $\hat{f}$ follows immediately from Part 2) of Theorem 3.9.

We proceed again by induction (II) on the integer $k(0 \leq k \leq N)$ such that there are at least $k$ complex manifolds among $\left\{D_{1}, \ldots, D_{N}\right\}$ which are biholomorphic to Euclidean domains.

For $k=N$ we are reduced to Theorem 2.3.

Suppose that Theorem A is true for the case where $k=k_{0}\left(1 \leq k_{0} \leq N\right)$. We have to discuss the case where $k=k_{0}-1$. Suppose without loss of generality that $D_{2}$ is not biholomorphic to an Euclidean domain.

For any $1 \leq j \leq N$ and $a_{j} \in A_{j} \cap A_{j}^{*}$, one fixes an open neighborhood $U_{a_{j}}$ of $a_{j}$ such that $U_{a_{j}}$ is biholomorphic to a domain in $\mathbb{C}^{d_{a_{j}}}$, where $d_{a_{j}}$ is the dimension of $D_{j}$ at $a_{j}$. For $1 \leq j \leq N$ and for any $0<\delta<1$, define

$$
\begin{aligned}
U_{a_{j}, \delta} & :=\left\{z_{j} \in U_{a_{j}}: \widetilde{\omega}\left(z_{j}, A_{j} \cap U_{a_{j}}, U_{a_{j}}\right)<\delta\right\}, \quad a_{j} \in A_{j} \cap A_{j}^{*}, \\
A_{j, \delta} & :=\bigcup_{a_{j} \in A_{j} \cap A_{j}^{*}} U_{a_{j}, \delta}, \\
D_{j, \delta} & :=\left\{z_{j} \in D_{j}: \widetilde{\omega}\left(z_{j}, A_{j}, D_{j}\right)<1-\delta\right\} .
\end{aligned}
$$

For every $a_{1} \in A_{1} \cap A_{1}^{*}$, consider the mapping $f_{a_{1}}$ provided by

$$
\begin{aligned}
f_{a_{1}}\left(z_{2}, \ldots, z_{N}\right):=f\left(a_{1}, z_{2}, \ldots, z_{N}\right) & \\
& \left(z_{2}, \ldots, z_{N}\right) \in \mathbb{X}\left(A_{2}, \ldots, A_{N} ; D_{2}, \ldots, D_{N}\right) .
\end{aligned}
$$

Observe that in virtue of the above formula and the hypothesis that $f \in \mathcal{O}_{s}(X, Z)$, $f_{a_{1}}$ satisfies the hypothesis of Theorem A for $(N-1)$-cross. Consequently, applying the hypothesis of induction (I), we obtain a unique mapping

$$
\hat{f}_{a_{1}} \in \mathcal{O}\left(\widehat{\mathbb{X}}\left(A_{2}, \ldots, A_{N} ; D_{2}, \ldots, D_{N}\right), Z\right)
$$

such that

$$
\begin{aligned}
& \hat{f}_{a_{1}}\left(z_{2}, \ldots, z_{N}\right)=f\left(a_{1}, z_{2}, \ldots, z_{N}\right) \\
& \left(z_{2}, \ldots, z_{N}\right) \in \mathbb{X}\left(A_{2} \cap A_{2}^{*}, \ldots, A_{N} \cap A_{N}^{*} ; D_{2}, \ldots, D_{N}\right) .
\end{aligned}
$$


For every $a_{2} \in A_{2} \cap A_{2}^{*}$, consider the mapping $f_{a_{2}}$ provided by

$$
\begin{aligned}
& f_{a_{2}}\left(z_{1}, z_{2}, z_{3}, \ldots, z_{N}\right):=f\left(z_{1}, z_{2}, z_{3}, \ldots, z_{N}\right), \\
& \left(z_{1}, z_{2}, z_{3}, \ldots, z_{N}\right) \in \mathbb{X}\left(A_{1}, A_{2} \cap U_{a_{2}}, A_{3}, \ldots, A_{N} ; D_{1}, U_{a_{2}}, D_{3}, \ldots, D_{N}\right) .
\end{aligned}
$$

Recall that $U_{a_{2}}$ is biholomorphic to an Euclidean domain, but $D_{2}$ is not so. Therefore, in virtue of the above formula and the hypothesis that $f \in \mathcal{O}_{s}(X, Z)$, we may apply the hypothesis of induction (II) to $f_{a_{2}}$. Consequently, one obtains a unique mapping

$$
\hat{f}_{a_{2}} \in \mathcal{O}\left(\widehat{\mathbb{X}}\left(A_{1}, A_{2} \cap U_{a_{2}}, A_{3}, \ldots, A_{N} ; D_{1}, U_{a_{2}}, D_{3}, \ldots, D_{N}\right), Z\right)
$$

such that

$$
\begin{aligned}
& \hat{f}_{a_{2}}\left(z_{1}, z_{2}, z_{3}, \ldots, z_{N}\right)=f\left(z_{1}, z_{2}, z_{3}, \ldots, z_{N}\right) \\
& \left(z_{1}, z_{2}, z_{3}, \ldots, z_{N}\right) \\
& \in \mathbb{X}\left(A_{1} \cap A_{1}^{*}, A_{2} \cap A_{2}^{*} \cap U_{a_{2}}, A_{3} \cap A_{3}^{*}, \ldots, A_{N} \cap A_{N}^{*} ; D_{1}, U_{a_{2}}, D_{3}, \ldots, D_{N}\right) .
\end{aligned}
$$

We need the following

Lemma 7.1. We keep the hypothesis of Theorem $A$ and the above notation. Then for any $a_{1} \in A_{1} \cap A_{1}^{*}, a_{2} \in A_{2} \cap A_{2}^{*}$, and any $0<\delta<\frac{1}{N}$,

$$
\begin{aligned}
\hat{f}_{a_{1}}\left(z_{2}, z_{3}, \ldots, z_{N}\right)=\hat{f}_{a_{2}}\left(a_{1}, z_{2}, z_{3}, \ldots, z_{N}\right), & \\
& \left(z_{2}, z_{3}, \ldots, z_{N}\right) \in U_{a_{2}, \delta} \times A_{3, \delta} \times \cdots \times A_{N, \delta} .
\end{aligned}
$$

Proof of Lemma 7.1. Let $a_{1}, a_{2}$ be as in the statement of Lemma 7.1. In virtue of (7.2)-(7.3),

$$
\begin{gathered}
\hat{f}_{a_{2}}\left(a_{1}, z_{2}, z_{3}, \ldots, z_{N}\right)=f\left(a_{1}, z_{2}, z_{3}, \ldots, z_{N}\right)=\hat{f}_{a_{1}}\left(z_{2}, z_{3}, \ldots, z_{N}\right), \\
\left(z_{2}, z_{3}, \ldots, z_{N}\right) \in \mathbb{X}\left(A_{2} \cap A_{2}^{*} \cap U_{a_{2}}, A_{3} \cap A_{3}^{*}, \ldots, A_{N} \cap A_{N}^{*} ; U_{a_{2}}, D_{3}, \ldots, D_{N}\right) .
\end{gathered}
$$

Consequently, applying Part 2) of Theorem 3.9 to $\hat{f}_{a_{1}}$ and $\hat{f}_{a_{2}}\left(a_{1}, \cdot\right)$ yields that

$$
\begin{aligned}
\hat{f}_{a_{1}}\left(z_{2}, z_{3}, \ldots, z_{N}\right)= & \hat{f}_{a_{2}}\left(a_{1}, z_{2}, z_{3}, \ldots, z_{N}\right), \\
\left(z_{2}, z_{3}, \ldots, z_{N}\right) & \in \widehat{\mathbb{X}}\left(A_{2} \cap U_{a_{2}}, A_{3}, \ldots, A_{N} ; U_{a_{2}}, D_{3}, \ldots, D_{N}\right) .
\end{aligned}
$$

Moreover, since $0<\delta<\frac{1}{N}$, it follows from (7.1), (6.2), and a straightforward computation that

$$
U_{a_{2}, \delta} \times A_{3, \delta} \times \cdots \times A_{N, \delta} \subset \widehat{\mathbb{X}}\left(A_{2} \cap U_{a_{2}}, A_{3}, \ldots, A_{N} ; U_{a_{2}}, D_{3}, \ldots, D_{N}\right) .
$$

This, combined with (7.4), implies the desired conclusion of the lemma. 
In the sequel we always suppose that $0<\delta<\frac{1}{N}$. In virtue of (7.3), Part 1) of Theorem 3.9 and Definition 6.3, we are able to collect the family of mappings

$$
\left(\left.\hat{f}_{a_{2}}\right|_{D_{1, N \delta}} \times U_{a_{2}, \delta} \times A_{3, \delta} \times \cdots \times A_{N, \delta}\right)_{a_{2} \in A_{2} \cap A_{2}^{*}}
$$

in order to obtain the collected mapping

$$
\tilde{\tilde{f}}_{\delta} \in \mathcal{O}\left(D_{1, N \delta} \times A_{2, \delta} \times \cdots \times A_{N, \delta}, Z\right)
$$

Let

$$
\begin{aligned}
X_{\delta} & :=\mathbb{X}\left(A_{1} \cap A_{1}^{*}, A_{2, \delta} \times \cdots \times A_{N, \delta} ; D_{1, N \delta}, \widehat{\mathbb{X}}\left(A_{2}, \ldots, A_{N} ; D_{2}, \ldots, D_{N}\right)\right), \\
\widehat{X}_{\delta} & :=\widehat{\mathbb{X}}\left(A_{1} \cap A_{1}^{*}, A_{2, \delta} \times \cdots \times A_{N, \delta} ; D_{1, N \delta}, \widehat{\mathbb{X}}\left(A_{2}, \ldots, A_{N} ; D_{2}, \ldots, D_{N}\right)\right) .
\end{aligned}
$$

In virtue of Lemma 7.1 and the construction (7.5), we are able to define a new mapping $\tilde{f}_{\delta}: X_{\delta} \longrightarrow Z$ as follows

$$
\tilde{f}_{\delta}(z):= \begin{cases}\tilde{\tilde{f}}_{\delta}(z), & z \in D_{1, N \delta} \times A_{2, \delta} \times \cdots \times A_{N, \delta}, \\ \hat{f}_{z_{1}}, & z \in\left(A_{1} \cap A_{1}^{*}\right) \times \widehat{\mathbb{X}}\left(A_{2}, \ldots, A_{N} ; D_{2}, \ldots, D_{N}\right),\end{cases}
$$

where $z=\left(z_{1}, \ldots, z_{N}\right) \in X_{\delta}$.

Using (7.7), (7.2) and (7.5), it can be readily checked that $\tilde{f}_{\delta} \in \mathcal{O}_{s}\left(X_{\delta}\right)$. In addition, using (7.6) we have that $X_{\delta} \cap X_{\delta}^{*}=X_{\delta}$. Consequently, for every $0<\delta<$ $\frac{1}{N}$, one applies Theorem 6.1 to $\tilde{f}_{\delta}$ and obtain a unique mapping $\hat{f}_{\delta} \in \mathcal{O}\left(\widehat{X}_{\delta}\right)$ such that

$$
\hat{f}_{\delta}=\tilde{f}_{\delta} \quad \text { on } X_{\delta} .
$$

Finally, gluing $\left(\hat{f}_{\delta}\right)_{0<\delta<\frac{1}{N}}$, we can define the desired extension mapping $\hat{f}$ by the formula

$$
\hat{f}:=\lim _{\delta \rightarrow 0} \hat{f}_{\delta} \quad \text { on } \widehat{X}
$$

Next, we argue as in the proof of Theorem 6.1. More precisely, one checks that the hypothesis of Lemma 6.5 is fulfilled with

$$
\begin{aligned}
\mathcal{D} & :=D_{1}, \\
\mathcal{G} & :=\widehat{\mathbb{X}}\left(A_{2}, \ldots, A_{N} ; D_{2}, \ldots, D_{N}\right), \\
\mathcal{A}_{\delta} & :=A_{1} \cap A_{1}^{*}, \\
\mathcal{B}_{\delta} & :=A_{2, \delta} \times \cdots \times A_{N, \delta}, \\
\mathcal{D}_{\delta} & :=D_{1, N \delta}, \\
\mathcal{G}_{\delta} & :=\widehat{\mathbb{X}}\left(A_{2}, \ldots, A_{N} ; D_{2}, \ldots, D_{N}\right),
\end{aligned}
$$

for $0<\delta<\frac{1}{N}$ 
To do this let

$$
\begin{aligned}
\Omega & :=\widehat{\mathbb{X}}\left(A_{2, \delta}, \ldots, A_{N, \delta} ; D_{2}, \ldots, D_{N}\right), \\
\Omega_{N \delta} & :=\left\{z^{\prime} \in \Omega: \widetilde{\omega}\left(z^{\prime}, A_{2, \delta} \times \cdots \times A_{N, \delta}, \Omega\right)<1-N \delta\right\} .
\end{aligned}
$$

Applying inequality (6.3), one gets

$$
\sum_{j=2}^{N} \widetilde{\omega}\left(z_{j}, A_{j}, D_{j}\right)<\sum_{j=2}^{N} \widetilde{\omega}\left(z_{j}, A_{j, \delta}, D_{j}\right)+N \delta<1, \quad z^{\prime}=\left(z_{2}, \ldots, z_{N}\right) \in \Omega_{N \delta}
$$

Hence, $\Omega_{N \delta} \subset \widehat{\mathbb{X}}\left(A_{2}, \ldots, A_{N} ; D_{2}, \ldots, D_{N}\right)$, which, in virtue of Part 2) of Proposition 3.5, implies that

$$
\begin{aligned}
\widetilde{\omega}\left(z^{\prime}, A_{2, \delta} \times \cdots \times A_{N, \delta}, \widehat{\mathbb{X}}\left(A_{2}, \ldots, A_{N} ; D_{2}, \ldots, D_{N}\right)\right) \\
\leq \widetilde{\omega}\left(z^{\prime}, A_{2, \delta} \times \cdots \times A_{N, \delta}, \Omega_{N \delta}\right), \quad z^{\prime} \in \Omega_{N \delta} .
\end{aligned}
$$

On the other hand, in virtue of Part 2) of Proposition 3.7, we have that $\widetilde{\omega}\left(z^{\prime}, A_{2, \delta} \times \cdots \times A_{N, \delta}, \Omega\right)=\sum_{j=2}^{N} \widetilde{\omega}\left(z_{j}, A_{j, \delta}, D_{j}\right), \quad z^{\prime}=\left(z_{2}, \ldots, z_{N}\right) \in \Omega$

By Part 4) of Proposition 3.5,

$$
\widetilde{\omega}\left(z^{\prime}, A_{2, \delta} \times \cdots \times A_{N, \delta}, \Omega_{N \delta}\right)=\frac{\widetilde{\omega}\left(z^{\prime}, A_{2, \delta} \times \cdots \times A_{N, \delta}, \Omega\right)}{1-N \delta}, \quad z^{\prime} \in \Omega_{N \delta} .
$$

This, combined with (7.10)-(7.11), implies that

$$
\widetilde{\omega}\left(z^{\prime}, A_{2, \delta} \times \cdots \times A_{N, \delta}, \widehat{\mathbb{X}}\left(A_{2}, \ldots, A_{N} ; D_{2}, \ldots, D_{N}\right)\right) \leq \frac{\sum_{j=2}^{N} \widetilde{\omega}\left(z_{j}, A_{j, \delta}, D_{j}\right)}{1-N \delta} .
$$

For every $z^{0}=\left(z_{1}^{0}, z^{0^{\prime}}\right) \in \widehat{X}$, let $\delta_{0}:=\frac{1-\sum_{j=1}^{N} \widetilde{\omega}\left(z_{j}^{0}, A_{j}, D_{j}\right)}{2 N}$ and fix an open neighborhood $U \times V$ of $z^{0}$ such that

$$
\sum_{j=1}^{N} \widetilde{\omega}\left(z_{j}, A_{j}, D_{j}\right)<\delta_{0}+\sum_{j=1}^{N} \widetilde{\omega}\left(z_{j}^{0}, A_{j}, D_{j}\right), \quad z=\left(z_{1}, z^{\prime}\right) \in U \times V .
$$


Then, using the latter estimate and (7.12) and Part 4) of Proposition 3.5, we see that

$$
\begin{gathered}
\widetilde{\omega}\left(z_{1}, A_{1} \cap A_{1}^{*}, D_{1, N \delta_{0}}\right)+\widetilde{\omega}\left(z^{\prime}, A_{2, \delta} \times \cdots \times A_{N, \delta}, \widehat{\mathbb{X}}\left(A_{2}, \ldots, A_{N} ; D_{2}, \ldots, D_{N}\right)\right) \\
\leq \frac{\sum_{j=1}^{N} \widetilde{\omega}\left(z_{j}^{0}, A_{j}, D_{j}\right)+\delta_{0}}{1-N \delta_{0}}<1 .
\end{gathered}
$$

for $z=\left(z_{1}, z^{\prime}\right) \in U \times V$ and $0<\delta \leq \delta_{0}$. Consequently, we are able to apply Lemma 6.5.

We complete the proof as follows. An immediate consequence of Lemma 6.5 and formula (7.9) is that $\hat{f} \in \mathcal{O}(\widehat{X}, Z)$. Moreover, by (7.2)-(7.3) and (7.6)-(7.9), and using the fact that $D_{1, N \delta} \nearrow D_{1}$ as $\delta \searrow 0$ (see (7.1)), we conclude that $\hat{f}=f$ on the following set

$$
\begin{aligned}
& \left(A_{1} \cap A_{1}^{*}\right) \times \mathbb{X}\left(A_{2} \cap A_{2}^{*}, \ldots, A_{N} \cap A_{N}^{*} ; D_{2}, \ldots, D_{N}\right) \\
& \bigcup D_{1} \times\left(A_{2} \cap A_{2}^{*}\right) \times \cdots \times\left(A_{N} \cap A_{N}^{*}\right) .
\end{aligned}
$$

Since this set is equal to $X \cap X^{*}$, it follows from Theorem 3.10 that the mapping $\hat{f}$ provided by formula (7.9) possesses all the desired properties. This completes induction (II) for $k=k_{0}-1$. Hence, the proofs of induction (II), induction (I) and then the first part of the theorem are finished.

\subsection{Proof of the estimate in Theorem $A$}

Following the work in [28] we divide this part into two steps.

Step 1. Proof of the inequality $|\hat{f}|_{\widehat{X}} \leq|f|_{X}$.

Proof of Step 1. In order to reach a contradiction assume that there is a point $z^{0} \in \widehat{X}$ such that $\left|\hat{f}\left(z^{0}\right)\right|>|f|_{X}$. Put $\alpha:=\hat{f}\left(z^{0}\right)$ and consider the function

$$
g(z):=\frac{1}{f(z)-\alpha}, \quad z \in X .
$$

Using the above assumption, we clearly have that $g \in \mathcal{O}_{s}(X, \mathbb{C})$. Hence by Subsection 8.1 , there is exactly one function $\hat{g} \in \mathcal{O}(\widehat{X}, \mathbb{C})$ with $\hat{g}=g$ on $X$. Therefore, by (7.13) we have on $X: g(f-\alpha) \equiv 1$. Thus $\hat{g}(\hat{f}-\alpha) \equiv 1$ on $\widehat{X}$. In particular,

$$
0=\hat{g}\left(z^{0}\right)\left(\hat{f}\left(z^{0}\right)-\alpha\right)=1,
$$

which is a contradiction. Hence the inequality $|\hat{f}|_{\widehat{X}} \leq|f|_{X}$ is proved. Thus Step 1 is complete.

Step 2. Proof of the inequality

$$
|\hat{f}(z)| \leq|f|_{A}^{1-\omega(z)}|f|_{X}^{\omega(z)} .
$$


Proof of Step 2. We prove (7.14) by induction on $N$. When $N=1$, then applying Theorem 3.11 to the plurisubharmonic function $z \in D_{1} \mapsto \log |\hat{f}(z)|$, (7.14) follows. Suppose that (7.14) is true for $N-1$. We would like to prove it for $N$. Fix an arbitrary point $z^{0}=\left(z_{1}^{0}, \ldots, z_{N}^{0}\right) \in \widehat{X}$. Let

$$
\delta:=\sum_{j=2}^{N} \widetilde{\omega}\left(z_{j}^{0}, A_{j}, D_{j}\right) .
$$

For any $a_{1} \in A_{1} \cap A_{1}^{*}$, we apply the hypothesis of induction to the function $\hat{f}_{a_{1}}$ and obtain the estimate

$$
\left|\hat{f}_{a_{1}}\left(z_{2}^{0}, \ldots, z_{N}^{0}\right)\right| \leq|f|_{A}^{1-\delta}|f|_{X}^{\delta} .
$$

In virtue of (7.7)-(7.9), we obtain that

$$
\hat{f}_{a_{1}}\left(z_{2}^{0}, \ldots, z_{N}^{0}\right)=\hat{f}\left(a_{1}, z_{2}^{0}, \ldots, z_{N}^{0}\right), \quad a_{1} \in A_{1} \cap A_{1}^{*} .
$$

This, combined with (7.16), implies that

$$
\left|\hat{f}\left(\cdot, z_{2}^{0}, \ldots, z_{N}^{0}\right)\right|_{A_{1} \cap A_{1}^{*}} \leq|f|_{A}^{1-\delta}|f|_{X}^{\delta} .
$$

On the other hand,

$$
\left|\hat{f}\left(\cdot, z_{2}^{0}, \ldots, z_{N}^{0}\right)\right|_{D_{1, \delta}} \leq|\hat{f}|_{\widehat{X}} \leq|f|_{X}
$$

where the latter estimate follows from Step 1.

Applying Theorem 3.11 to the function $\log \left|\hat{f}\left(\cdot, z_{2}^{0}, \ldots, z_{N}^{0}\right)\right|_{D_{1, \delta}}$, and taking (7.17) and (7.18) into account, we obtain

$$
\begin{aligned}
\left|\hat{f}\left(z^{0}\right)\right| & \leq\left|\hat{f}\left(\cdot, z_{2}^{0}, \ldots, z_{N}^{0}\right)\right|_{A_{1} \cap A_{1}^{*}}^{1-\widetilde{\omega}\left(z_{1}^{0}, A_{1} \cap A_{1}^{*}, D_{1, \delta}\right)}\left|\hat{f}\left(\cdot, z_{2}^{0}, \ldots, z_{N}^{0}\right)\right|_{D_{1, \delta}}^{\widetilde{\omega}\left(z_{1}^{0}, A_{1} \cap A_{1}^{*}, D_{1, \delta}\right)} \\
& =|f|_{A}^{1-\omega\left(z^{0}\right)}|f|_{X}^{\omega\left(z^{0}\right)}
\end{aligned}
$$

where the equality follows from (7.15) and the identity $\widetilde{\omega}\left(z_{1}^{0}, A_{1} \cap A_{1}^{*}, D_{1, \delta}\right)=$ $\frac{\widetilde{\omega}\left(z_{1}^{0}, A_{1} \cap A_{1}^{*}, D_{1}\right)}{1-\delta}$ (by Part 4) of Proposition 3.5). Hence estimate (7.14) for the point $z^{0}$ is proved. Since $z_{0}$ is an arbitrary point in $\widehat{X}$, (7.14) follows, and the proof of the estimate in Theorem A is thereby finished.

Combining the result of Subsections 7.1 and 7.2, Theorem A follows. 
Finally, we conclude the article by some remarks and open questions.

1. Recent development in the theory of separately analytic mappings is characterized by cross theorems with pluripolar singularities and boundary cross theorems. The most general results are contained in some articles of Jarnicki and Pflug (see $[12,13,14])$ and in recent works of Pflug and the author (see [28, 29]). The question naturally arises whether one can generalize these results in the context of mappings defined on complex manifolds with values in a complex analytic space. We postpone this issue to an ongoing work.

2. Is Theorem $A$ in the case where $Z:=\mathbb{C}$ optimal? In other words, is the open set $\widehat{X}$ always the envelope of holomorphy for separately holomorphic functions defined on $X$ ?

\section{References}

[1] O. Alehyane et J. M. HeCART Propriété de stabilité de la fonction extrémale relative, preprint, (1999).

[2] K. AdACHI, M. SuzUKI and M. Yoshida, Continuation of holomorphic mappings with values in a complex Lie group, Pacific J. Math. 47 (1973), 1-4.

[3] O. AlehyANE et A. ZERIAHI, Une nouvelle version du théorème d'extension de Hartogs pour les applications séparément holomorphes entre espaces analytiques, Ann. Polon. Math. 76 (2001), 245-278.

[4] E. Bedford, The operator $\left(d d^{c}\right)^{n}$ on complex spaces, Semin. P. Lelong - H. Skoda, Analyse, Années 1980/81, Lect. Notes Math. 919 (1982), 294-323.

[5] E. BEDFORD and B. A. TAYLOR, A new capacity for plurisubharmonic functions, Acta Math. 149 (1982), 1-40.

[6] A. Edigarian, Analytic discs method in complex analysis, Dissertationes Math. 402 (2002).

[7] A. Edigarian and E. A. POletsky, Product property of the relative extremal function, Bull. Polish Acad. Sci. Math. 45 (1997), 331-335.

[8] F. HARTOGS, Zur Theorie der analytischen Funktionen mehrer unabhängiger Veränderlichen, insbesondere über die Darstellung derselben durch Reihen, welche nach Potenzen einer Veränderlichen fortschreiten, Math. Ann. 62 (1906), 1-88.

[9] B. JosEFSON, On the equivalence between polar and globally polar sets for plurisubharmonic functions on $\mathbb{C}^{n}$, Ark. Mat. 16 (1978), 109-115.

[10] S. M. IVASHKOVICH, The Hartogs phenomenon for holomorphically convex Kähler manifolds, Math. USSR-Izv. 29 (1997), 225-232.

[11] M. JARniCKI and P. Pflug, "Extension of Holomorphic Functions", de Gruyter Expositions in Mathematics 34, Walter de Gruyter, 2000.

[12] M. JARNICKI and P. PFLUG, An extension theorem for separately holomorphic functions with analytic singularities, Ann. Polon Math. 80 (2003), 143-161.

[13] M. JARNICKI and P. PFLUG, An extension theorem for separately holomorphic functions with pluripolar singularities, Trans. Amer. Math. Soc. 355 (2003), 1251-1267.

[14] M. JARNICKI and P. PFLUG, An extension theorem for separately meromorphic functions with pluripolar singularities, Kyushu J. of Math., 57 (2003), 291-302.

[15] M. KLIMEK, "Pluripotential theory", London Mathematical society monographs, Oxford Univ. Press., 6, 1991. 
[16] N. V. KHUE and N. H. ThANH, Locally bounded holomorphic functions and the mixed Hartogs theorem, Southeast Asian Bull. Math. 23 (1999), 643-655.

[17] F. LÁrusson and R. Sigurdsson, Plurisubharmonic functions and analytic discs on manifolds, J. Reine Angew. Math. 501 (1998), 1-39.

[18] NGUyên Thanh VÂn, Separate analyticity and related subjects, Vietnam J. Math. 25 (1997), 81-90.

[19] NGUYÊn Thanh VÂN, Note on doubly orthogonal system of Bergman, In: "Linear Topological Spaces and Complex Analysis" 3 (1997), 157-159.

[20] Ph. NoverraZ, Fonctions plurisousharmoniques et analytiques dans les espaces vectoriels topologiques, Ann. Inst. Fourier Grenoble 19 (1969), 419-493.

[21] NGUyÊn Thanh VÂn and J. SiciaK, Fonctions plurisousharmoniques extrémales et systèmes doublement orthogonaux de fonctions analytiques, Bull. Sci. Math. 115 (1991), 235-244.

[22] NguyÊn Thanh VÂn et A. Zeriahi, Familles de polynômes presque partout bornées, Bull. Sci. Math. 107 (1983), 81-89.

[23] NGUyên Thanh VÂN et A. ZeRIAhI, Une extension du théorème de Hartogs sur les fonctions séparément analytiques, In: "Analyse Complexe Multivariable, Récents Développements", A. Meril (ed.), EditEl, Rende, 1991, 183-194.

[24] NGuyên Thanh VÂn et A. Zeriahi, Systèmes doublement orthogonaux de fonctions holomorphes et applications, Banach Center Publ. 31, Inst. Math., Polish Acad. Sci. (1995), 281-297.

[25] P. Pflug, Extension of separately holomorphic functions-a survey 1899-2001, Ann. Polon. Math. 80 (2003), 21-36.

[26] E. A. Poletsky, Plurisubharmonic functions as solutions of variational problems, In: "Several complex variables and complex geometry", Proc. Summer Res. Inst., Santa Cruz/CA (USA) 1989, Proc. Symp. Pure Math. 52, Part 1 (1991), 163-171.

[27] E. A. Poletsky, Holomorphic currents, Indiana Univ. Math. J. 42 (1993), 85-144.

[28] P. Pflug and V.-A. NGUYÊN, A boundary cross theorem for separately holomorphic functions, Ann. Polon. Math. 84 (2004), 237-271.

[29] P. Pflug and V.-A. NGuYÊN, Generalization of Drużkowski's and Gonchar's "Edge-ofthe-Wedge" Theorems, preprint 2004, available at arXiv:math.CV/0503326.

[30] P. PFLUG and V.-A. NGUYÊN, Envelope of holomorphy for boundary cross sets, preprint 2005.

[31] T. RANSFORD, "Potential theory in the complex plane", London Mathematical Society Student Texts 28, Cambridge: Univ. Press., 1995.

[32] J. P. RosAY, Poletsky theory of disks on holomorphic manifolds, Indiana Univ. Math. J. 52 (2003), 157-169.

[33] A. Sadullaev, Plurisubharmonic measures and capacities on complex manifolds, Russian Math. Surveys 36 (1981), 61-119.

[34] B. SHIFFMAN, Extension of holomorphic maps into Hermitian manifolds, Math. Ann. 194 (1971), 249-258.

[35] I. SHImOdA, Notes on the functions of two complex variables, J. Gakugei Tokushima Univ. 8 (1957), 1-3.

[36] J. SICIAK, Analyticity and separate analyticity of functions defined on lower dimensional subsets of $\mathbb{C}^{n}$, Zeszyty Nauk. Univ. Jagiello. Prace Mat. Zeszyt 13 (1969), 53-70.

[37] J. SiCIAK, Separately analytic functions and envelopes of holomorphy of some lower dimensional subsets of $\mathbb{C}^{n}$, Ann. Polon. Math. 22 (1970), 145-171.

[38] T. TERADA, Sur une certaine condition sous laquelle une fonction de plusieurs variables complexes est holomorphe, Publ. Res. Inst. Math. Sci. 2 (1967), 383-396.

[39] V. P. ZAHARIUTA, Separately analytic functions, generalizations of the Hartogs theorem and envelopes of holomorphy, Math. USSR-Sb. 30 (1976), 51-67. 
[40] A. ZERIAHI, Comportement asymptotique des systèmes doublement orthogonaux de Bergman: Une approche élémentaire, Vietnam J. Math. 30 (2002), 177-188.

[41] H. Wu, Normal families of holomorphic mappings, Acta Math. 119 (1967), 193-233.

Max-Planck-Institut für Mathematik

Vivatsgasse 7

D-53111 Bonn, Germany

vietanh@mpim-bonn.mpg.de 